\title{
A Unified Framework for Design and Analysis of Networked and Quantized Control Systems
}

\author{
Dragan Nešić, Fellow, IEEE, and Daniel Liberzon, Senior Member, IEEE
}

\begin{abstract}
We generalize and unify a range of recent results in quantized control systems (QCS) and networked control systems (NCS) literature and provide a unified framework for controller design for control systems with quantization and time scheduling via an emulation-like approach. A crucial step in our proofs is finding an appropriate Lyapunov function for the quantization/ time-scheduling protocol which verifies its uniform global exponential stability (UGES). We construct Lyapunov functions for several representative protocols that are commonly found in the literature, as well as some new protocols not considered previously. Our approach is flexible and amenable to further extensions which are briefly discussed.
\end{abstract}

Index Terms-Networked control systems (NCS), quantized control systems (QCS).

\section{INTRODUCTION}

C ONTROL of systems over band-limited channels is currently attracting a lot of attention in the control community due to a range of emerging control applications. In such systems, certain control loops contain channels in which only a finite amount of data can be transferred at any transmission instant, and this leads to communication constraints that cannot be ignored in the controller design. Currently, there are two main approaches to modelling band-limited communication channels in control loops: i) the channel is digital and due to the finite word length effects only a finite number of bits can be transmitted over the channel at any transmission instant. The main issue in control (stabilization) of systems with such channels is that of quantization and we use the term quantized control systems ( $Q C S$ ) to denote systems exhibiting this feature (see, for instance, [1], [4], [17], [26] and references cited therein); ii) the channel is a serial bus and only a subset of sensors and/or actuators can transmit their data over the channel at each transmission instant. In this case, the sensor/actuator data is transmitted in packets and typically the quantization effects are ignored in the analysis (see [8], [9], [11], [19], [24], [27] and references therein). The main issue in this class of systems is time scheduling of transmissions of various signals in the system and these systems are often referred to in the literature as networked control systems (NCS).

Manuscript received September 29, 2007; revised June 10, 2008. Current version published April 08, 2009. This work was supported by the Australian Research Council under the Discovery Grants and Australian Professorial Fellow schemes and by National Science Foundation (NSF) Grant ECCS-0701676. Recommended by Associate Editor J. P. Hespanha.

D. Nešić is with the Department of Electrical and Electronic Engineering, University of Melbourne, Victoria 3000, Australia (e-mail: d.nesic@ee.unimelb.edu.au).

D. Liberzon is with the Coordinated Science Laboratory, University of Illinois at Urbana-Champaign, Urbana, IL 61801 USA (e-mail: liberzon@uiuc.edu).

Digital Object Identifier 10.1109/TAC.2009.2014930
These two modelling approaches evolved separately in the literature with little cross-referencing or cross-fertilization. However, a very similar controller design approach has been proposed for both QCS and NCS and this approach consists of the following steps: i) ignore the communication constraints of the channels (quantization or time scheduling) and design a controller using the classical techniques; ii) design/choose a particular quantizer or partition of the sensor/actuator vector, as well as an algorithm (protocol) that governs the quantization or time-scheduling during the operation of the system; iii) determine a sufficiently small upper bound on the inter-transmission intervals, the so-called maximal allowable transmission interval (MATI), that guarantees the stability of the system. For instance, this controller design approach was proposed in [27] for NCS and it was used in [13] for QCS. We note that this approach is a natural generalization of the emulation approach to controller design for sampled-data systems, see e.g., [2].

We mention that, while there have been no systematic attempts to unify formulations and techniques from the NCS and QCS literature, some specific designs combining quantization and time scheduling have been proposed, for instance, in [6], [16], [17], [25]. We believe that this fact confirms the need for developing a general framework encompassing a large class of such protocols, which is the goal of this work. In the sequel, we refer to systems that combine time scheduling with quantization as networked and quantized control systems (NQCS).

The main purpose of this paper is to unify the controller design approaches mentioned above for QCS and NCS which naturally leads to the following contributions: i) We provide a unified framework for the emulation design approach which covers general NQCS and which is flexible, general and amenable to further extensions and modifications; ii) Our unified controller emulation framework brings two seemingly unrelated areas (QCS and NCS) under one umbrella and facilitates a cross-fertilization between them. For instance, we show that the notion of uniformly globally exponentially stable (UGES) protocols that was introduced in the NCS literature (see [19]) has a natural interpretation in QCS. We believe that this is the first time in the literature that this connection is made. Moreover, the small-gain theorems used in stability proofs of NCS in [19] provide new insights for QCS considered, for instance, in [1] and [13]; iii) We show that many existing protocols (for NCS or QCS) as well as new protocols (for NQCS) are UGES in the sense of our definition, and we provide constructions of Lyapunov functions for these protocols in Section IV. On one hand, this analysis provides a completely new view of "box" and "zoom" protocols found in the quantization literature. On the other hand, this leads to an analysis of genuinely new protocols for NQCS that combine quantization and time scheduling, such as the TOD with "box" 
protocols considered in Section IV-C. (iv) An outcome of our unified approach is a range of emulation-type results stated in Section V which provide analytic bounds on the MATI that guarantee stability of various classes of NCS, QCS and NQCS with various protocols. We note that these bounds are essential in applying the emulation procedure and they provide an insight into how the controller and the protocol affect the stability of the overall closed-loop system.

The paper is organized as follows. Section II contains the mathematical preliminaries and background results that are adapted from [19]. In Section III we illustrate that models of a range of NCS and QCS that arise in the literature, as well as NQCS not considered previously, are special cases of a particular class of systems with jumps to which our main results apply. We do not attempt to be exhaustive in this section and there may be other classes of systems that fit our framework. We show that the jump equation in this model depends solely on the network protocol. In Section IV, which contains our main technical contributions, we show that numerous protocols found in NCS, QCS and some new protocols for NQCS that we introduce are Lyapunov UGES in the sense defined in Section II. In Section V we combine the results from preceding sections to state explicit bounds on MATI that guarantee stability of the closed loop with emulated controllers in various situations. Some possible generalizations and conclusions are presented in the last section. Several auxiliary results are stated and proved in Appendix.

\section{PReliminaries AND BACKGRoUnd Results}

$\mathbb{R}$ and $\mathbb{N}$ denote, respectively, the sets of real and natural numbers. $\mathbb{R}_{\geq 0}$ denotes the set of non-negative real numbers. Given $t \in \mathbb{R}_{\geq 0}$ and a piecewise continuous function $f: \mathbb{R} \rightarrow \mathbb{R}^{n}$, we use the notation $f\left(t^{+}\right):=\lim _{s \backslash t} f(s)$. The Euclidean norm on $\mathbb{R}^{n}$ is denoted by $|\cdot|$; sometimes we will also use the infinity norm $\|x\|_{\infty}:=\max \left\{\left|x_{i}\right|: 1 \leq i \leq n\right\}$ on $\mathbb{R}^{n}$. The corresponding induced matrix norms are denoted respectively as $\|\cdot\|$ and $\|\cdot\|_{\infty}$. Given a piecewise continuous signal $\varphi: J \rightarrow \mathbb{R}^{n}$, where $J$ is a subinterval of $\left[t_{0}, \infty\right)$, we define its $\mathcal{L}_{\infty}$ norm as follows: $\|\varphi\|_{\infty}:=\sup _{s \in J}|\varphi(s)|$. If $\varphi(\cdot)$ is defined on $\left[t_{0}, \infty\right)$ and there exists $K \geq 0$ such that $\|\varphi\|_{\infty} \leq K$, then we write $\varphi \in \mathcal{L}_{\infty}$. A function $\gamma: \mathbb{R}_{>0} \rightarrow \mathbb{R}_{>0}$ is said to be of class $\mathcal{K}_{\infty}$ if it is continuous, zero at zero, strictly increasing and unbounded. A function $\beta: \mathbb{R}_{\geq 0} \times \mathbb{R}_{\geq 0} \rightarrow \mathbb{R}_{\geq 0}$ is said to be of class $\mathcal{K} \mathcal{L}$ if for each $s \geq 0$ the function $\beta(s, \cdot)$ is decreasing to zero and for each fixed $t \geq 0$ the function $\beta(\cdot, t)$ is of class $\mathcal{K}$. A function $\beta$ is said to be of class exp- $\mathcal{K} \mathcal{L}$ if there exist $K, c>0$ such that $\beta(s, t)=K \exp (-c t) s$. To shorten notation, we often use $(x, y):=\left(\begin{array}{ll}x^{T} & y^{T}\end{array}\right)^{T}$. We write $\operatorname{diag}\left\{A_{1}, \ldots, A_{\ell}\right\}$ for the (block-)diagonal matrix with the indicated elements on the diagonal and zeros elsewhere. For the systems we consider in this paper, a monotonically increasing sequence of times $t_{i} \in \mathbb{R}_{\geq 0}$ is given where $i \in \mathbb{N}$ and $t_{0}=0$. Moreover, we assume that there exist $\varepsilon>0$ and $\tau>\varepsilon$ such that ${ }^{1}$

$$
\varepsilon \leq t_{i}-t_{i-1} \leq \tau \quad \forall i \in \mathbb{N} .
$$

\footnotetext{
${ }^{1}$ The bound $\varepsilon \leq t_{i+1}-t_{i}$ guarantees that the system does not exhibit Zeno solutions. This assumption can be relaxed by requiring that the sequence $t_{i}$ be strictly increasing and $\lim _{i \rightarrow \infty} t_{i}=\infty$.
}

We set the stage by outlining the emulation approach and recalling a result that follows from [19]. The emulation approach was pioneered in [27] for NCS without disturbances and further developed in [19] for systems with disturbances. The same approach was pursued in [13] and elsewhere for QCS. Here we propose to apply this approach to NQCS. The first step in the emulation approach is to design a controller for a given plant ignoring the network (i.e., quantization and/or time scheduling). Namely, given a plant

$$
\dot{x}=\tilde{f}(t, x, u)
$$

one first designs a "nominal" controller

$$
u=k(t, x) .
$$

However, in the presence of time scheduling and/or quantization, the state $x$ is not directly available for control. So, one instead closes the loop with the controller

$$
u=k(t, \hat{x})
$$

where $\hat{x}$ is an estimate of $x$ generated using quantized state values transmitted over the network. In Section III we will explain how $\hat{x}$ is to be generated in various cases.

To model the systems arising in this way, we consider the following class of systems with jumps which is central to our approach:

$$
\begin{aligned}
\dot{x} & =f(t, x, z) \quad \forall t \in\left[t_{i-1}, t_{i}\right] \\
\dot{z} & =g(t, x, z) \quad \forall t \in\left[t_{i-1}, t_{i}\right] \\
z\left(t_{i}^{+}\right) & =h\left(i, x\left(t_{i}\right), z\left(t_{i}\right)\right)
\end{aligned}
$$

where $x \in \mathbb{R}^{n_{x}}, z \in \mathbb{R}^{n_{z}}, t_{i}$ satisfy (1) and $0<\varepsilon<\tau$. Here (5) gives the closed-loop plant dynamics, while $z$ includes the network-induced error variables as well as some other auxiliary variables needed to implement the quantization procedure. We will show in Section III that several known classes of NCS and QCS, as well as their NQCS generalizations, can be written in the above form. Our approach will apply to all of these different systems in a unified manner. We adopt terminology from [27] and refer to $\tau$ as the maximum allowable transmission interval (MATI). We note that even for the NCS case, the above model is more general than the one considered in [19] because the jump equation is allowed to depend explicitly on $x$. For a precise definition of solutions to such systems, please refer to [19].

We can assume that the system

$$
\dot{x}=f(t, x, 0)
$$

is stable in an appropriate sense. Note that (8) is the "nominal" closed-loop system without the quantization and/or time scheduling (i.e., no bandwidth limitations), obtained from (5) by setting $z \equiv 0$. Indeed, we will see in Section III that (8) is the same as (2), (3). However, we need to explicitly characterize robustness of the plant with respect to $z$ viewed as an exogenous input. We do this by employing the following (standard) notions; see, e.g., [22] for time-invariant systems and [5] for time-varying systems.

Definition 1: The system $\dot{x}=f(t, x, z)$ is input-to-state stable (ISS) from $z$ to $x$ if there exist functions $\kappa \in \mathcal{K}$ and 
$\beta \in \mathcal{K} \mathcal{L}$ such that for all $t_{0} \geq 0, x_{0} \in \mathbb{R}^{n_{x}}, z \in \mathcal{L}_{\infty}$ and each corresponding solution $x(\cdot)$, we have that

$$
|x(t)| \leq \beta\left(\left|x_{0}\right|, t-t_{0}\right)+\kappa\left(\|z\|_{\infty}\right) \quad \forall t \geq t_{0} \geq 0 .
$$

If the system is ISS with $\kappa(s)=\gamma \cdot s, \gamma \geq 0$ a linear function and

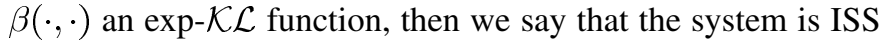
with a linear gain and an exp- $\mathcal{K} \mathcal{L}$ function. A system with state $x$ and no input $(z \equiv 0)$ is uniformly globally asymptotically stable (UGAS) if for all $x_{0} \in \mathbb{R}^{n_{x}}$ and all corresponding solutions $x(\cdot)$, we have that

$$
|x(t)| \leq \beta\left(\left|x_{0}\right|, t-t_{0}\right) \quad \forall t \geq t_{0} \geq 0 .
$$

The system is uniformly globally exponentially stable (UGES) if the above holds with a class exp- $\mathcal{K} \mathcal{L}$ function $\beta$.

Motivated by the results in [19], we refer to the jump equation (7) as the network "protocol". Our main results in Section V are presented for a large class of "UGES protocols" that are characterized in Definition 2 stated below. To define this class of protocols, we introduce an auxiliary discrete-time system

$$
z^{+}=h(i, x, z) \quad i \in \mathbb{N}
$$

where $h$ comes from (7); here $z^{+}$is a shorthand for $z(i+1)$ while the variables on the right-hand side are evaluated at time step $i$. We refer to (9) as a discrete-time system induced by the protocol (7), or simply as a protocol. Note that we treat $x$ in the above equation as an input. Central to this paper is the following class of protocols ${ }^{2}$ :

Definition 2: We say that the protocol (7) is uniformly globally exponentially stable (UGES) with Lyapunov function $W$ if there exist $W: \mathbb{N} \times \mathbb{R}^{n_{z}} \rightarrow \mathbb{R}_{\geq 0}, \rho \in[0,1)$ and $a_{1}, a_{2}>0$ such that we have

$$
\begin{aligned}
a_{1}|z| & \leq W(i, z) \leq a_{2}|z| \\
W(i+1, h(i, x, z)) & \leq \rho W(i, z)
\end{aligned}
$$

for all $i \in \mathbb{N}, x \in \mathbb{R}^{n_{x}}$ and all $z \in \mathbb{R}^{n_{z}}$.

In other words, the protocol (7) is UGES with Lyapunov function $W$ if $W$ is a UGES Lyapunov function for the system (9). Sometimes we will simply refer to such protocols as Lyapunov UGES or just UGES protocols when the Lyapunov function $W$ is not being specified. Section IV contains many examples of various time-scheduling and quantization protocols, as well as proofs of their UGES properties. Linear bounds are used in (10) instead of more standard quadratic ones in order to streamline the calculations. This does not introduce a loss of generality since, given a function with quadratic bounds, we can just take its square root in order to obtain another function satisfying (10) (we will see such constructions in Section IV).

The following result is a corollary of main results in [19] and in Section V it will be used to state a range of new emulation results for classes of NCS, QCS and NQCS that are introduced in Section III.

Theorem 1: Consider the system (5)-(7) with (1). Suppose that the following conditions hold:

\footnotetext{
${ }^{2}$ This definition was first used in [19] for a smaller class of protocols of the form $z^{+}=h(i, z)$ whose right hand side is independent of $x$. However, with the definition we use here, all main results in [19] still hold for the system (5)-(7).
}

i) System (5) is ISS from $z$ to $x$ with linear gain $\kappa(s)=\gamma \cdot s$, $\gamma \geq 0$

ii) Inequalities (10), (11) hold, i.e., the protocol (7) is Lyapunov UGES with a Lyapunov function $W(i, z)$;

iii) For some $L, c \geq 0$, we have that $W$ from item ii) and $g$ in (6) satisfy

$$
\left\langle\frac{\partial W(i, z)}{\partial z}, g(t, x, z)\right\rangle \leq L W(i, z)+c|x|
$$

for almost all $z \in \mathbb{R}^{n_{z}}, x \in \mathbb{R}^{n_{x}}$ and $t \geq 0$.

iv) MATI in (1) satisfies $\tau \in\left(\varepsilon, \tau^{*}\right)$ where

$$
\tau^{*}:=\frac{1}{L} \ln \left(\frac{L+c \gamma / a_{1}}{\rho L+c \gamma / a_{1}}\right)
$$

$\varepsilon \in\left(0, \tau^{*}\right)$ is arbitrary, $L$ and $c$ come from (12), $\gamma$ from item i), $a_{1}$ from (10), and $\rho$ from (11).

Then, the system (5)-(7) is UGAS. Moreover, if the system (5) is ISS from $z$ to $x$ with linear gain and exp- $\mathcal{K} \mathcal{L}$ function $\beta$, then the system (5)-(7) is UGES.

The main idea behind the proof of this result is to use items ii)-iv) to show that the $z$-subsystem is ISS with respect to $x$, and then use item i) and invoke an ISS small-gain theorem (see, e.g., [10]) to prove stability of the overall closed-loop system; the MATI bound (13) ensures that the small-gain condition holds. Theorem 1 is general enough to cover many cases of interest and illustrate the unifying nature of our approach. We emphasize, however, that using results of [19] one can state a range of more general or alternative stability results, such as results based on $\mathcal{L}_{p}$ stability and an appropriate small-gain theorem, which are omitted for space reasons.

\section{SYSTEM MODELS}

In this section we demonstrate that models of various NCS and QCS that were previously considered in the literature, as well as NQCS that were not considered in the literature, can be written in the form (5)-(7). This modelling framework is central to our approach as it allows us to unify, generalize, and compare many results in the literature. Indeed, we first show that models of NCS considered in [19], [27] and references cited therein are a special case of (5)-(7). This fact was already observed in [19] so we just quickly repeat the necessary steps for completeness and to illustrate the unifying nature of our results. Second, we show that models of QCS with "box" quantization protocols considered in [13], [14], [26] and the references therein are a special case of (5)-(7). As far as we are aware, this is the first time that QCS are modelled in this particular form. Third, we show that one can combine general time-scheduling and "box" quantization protocols that are respectively used in NCS and QCS mentioned above in order to obtain a more general NQCS model that is still a special case of (5)-(7). Examples of such schemes can be found in [6], [17], and [16]. Finally, we show that one can combine "zoom" protocols considered in [15] with the time-scheduling protocols in [19] to obtain a model that is again a special case of (5)-(7). These examples illustrate the unifying nature of our approach and we emphasize that the same framework could be useful in other situations not considered here. 
In order to simplify the presentation, we consider static state feedback controllers and plants without disturbances, and we assume that only the state is sent over the network. Each of these simplifying assumptions can be relaxed. For instance, the NCS results in [27] were presented for dynamic output feedback controllers where both the control input and plant output are sent over the network. These results were further generalized in [19] to cover general systems with exogenous disturbances. Output quantization was addressed in [13], and the QCS framework developed in [12] covers both input and output quantization. Exogenous disturbances in QCS are the subject of [15]. However, since no unified treatment of NCS and QCS has been attempted in the literature, we choose to avoid these more challenging scenarios in order to make our models more transparent.

\section{A. NCS}

For the purpose of comparison and to illustrate the unifying nature of our results, we reproduce the class of models for NCS considered in [19], [27] and show that it is a special case of (5)-(7). In this and Sections III-B-D, we always assume that (1) holds. Consider a general nonlinear plant (2) where $x \in \mathbb{R}^{n}$. It was proposed in [27] to first design a "nominal" controller (3) ignoring the effects of the network, so that the closed-loop system (2), (3) is stable in an appropriate sense. The designed controller is then implemented over the network in the following manner. We assume that the vector $x$ is partitioned into $\ell, 1 \leq$ $\ell \leq n$ different subvectors enumerated from 1 to $\ell$, i.e., $x=$ $\left(x_{1}, x_{2}, \ldots, x_{\ell}\right)$. We refer to the $i$ th subvector as the $i$ th "node". At each transmission time $t_{i}$, the protocol gives access to the network to one of the nodes $i \in\{1,2, \ldots, \ell\}$. We consider general nonlinear NCS of the following form:

$$
\begin{aligned}
\dot{x} & =\tilde{f}(t, x, k(t, \hat{x})) & \forall t \in\left[t_{i-1}, t_{i}\right] \\
\dot{\hat{x}} & =0 & \forall t \in\left[t_{i-1}, t_{i}\right] \\
\hat{x}\left(t_{i}^{+}\right) & =x\left(t_{i}\right)+h\left(i, e\left(t_{i}\right)\right) &
\end{aligned}
$$

where $\hat{x}$ is the vector of most recently transmitted plant state values via the network, $e:=\hat{x}-x$ is the network-induced error, and we closed the loop with the controller (4). We assume that $\hat{x}$ is held constant between the transmission instants (i.e., we use a zero-order hold in each node). The function $h$ is typically such that, if the $j$ th node gets access to the network at some transmission time $t_{i}$, the corresponding part of the error vector is reset to zero. However, neither of these assumptions (zero-order hold and resetting the error to zero) is necessary in the present framework, and both will be relaxed later in order to handle quantization effects. Rewriting the system in $(x, e)$ coordinates, we obtain the following NCS model:

$$
\begin{aligned}
\dot{x} & =f(t, x, e) \quad \forall t \in\left[t_{i-1}, t_{i}\right] \\
\dot{e} & =g(t, x, e) \quad \forall t \in\left[t_{i-1}, t_{i}\right] \\
e\left(t_{i}^{+}\right) & =h\left(i, e\left(t_{i}\right)\right)
\end{aligned}
$$

where

$$
f(t, x, e):=\tilde{f}(t, x, k(t, x+e)) ; \quad g(t, x, e):=-f(t, x, e) .
$$

The system (15)-(17) has exactly the same form as (5)-(7) if we think of $e$ in (16), (17) as $z$ in (6), (7). The error vector $e$ models the effects of the network and, since we assumed that NCS has $\ell$ nodes, it can be partitioned as $e=\left(e_{1}, e_{2}, \ldots, e_{\ell}\right)$. We refer to the jump equation (17) as a time-scheduling protocol. In [19], time-scheduling protocols with the maps $h$ of the following form were considered:

$$
h(i, e)=(I-\Psi(s)) e
$$

where $s=s(i, e): \mathbb{N} \times \mathbb{R}^{n} \rightarrow\{1, \ldots, \ell\}$ is some scheduling function

$$
\Psi(s)=\operatorname{diag}\left\{\delta_{1 s} I_{n_{1}}, \ldots, \delta_{\ell s} I_{n_{\ell}}\right\}
$$

$\ell$ is the number of nodes, $\delta_{i j}$ is the standard Kronecker delta, and $I_{n_{j}}$ are identity matrices of dimension $n_{j}$, with $\sum_{j=1}^{\ell} n_{j}=$ $n$. The above model of the protocol assumes that if the node $j$ is transmitted at time $t_{i}$ over the network, then $e_{j}\left(t_{i}^{+}\right)=0$. Examples of this class of protocols are given in Section IV-A.

\section{B. $Q C S$}

We now consider the model for QCS described in [13] for linear plants and in [14] for nonlinear plants. Models considered elsewhere (e.g., [6], [17], [26]) are very similar. However, in the QCS literature these models have previously not been written in the way we do it here.

Let the plant be given by (2), where $x \in \mathbb{R}^{n}$. We assume that a nominal feedback law (3) is given. When the state is quantized, this feedback law is not implementable. Instead, the control law will depend not on the state $x$ but on its estimate, $\hat{x}$, which we generate as follows. In between the times $t_{i}$, we let

$$
\dot{\hat{x}}=\tilde{f}(t, \hat{x}, u)
$$

which is a "copy" of the plant dynamics. The initial condition can be arbitrary, e.g., $\hat{x}\left(t_{0}\right)=0$. At each $t_{i}$, we reset $\hat{x}$ to a new value obtained from the quantized measurement. The quantizer, $q$, is defined by three parameters: an integer $N>1$ (a given constant which defines the number of quantization levels), $\hat{x} \in \mathbb{R}^{n}$ (the current state estimate), and $\xi \in \mathbb{R}_{>0}$ (an auxiliary variable which defines the size of the quantization regions). Consider a hypercubic box centered at $\hat{x}$ with edges $2 \xi$ and divide it into $N^{n}$ equal smaller sub-boxes ( $N$ in each dimension), numbered from 1 to $N^{n}$ in some specific way. ${ }^{3}$ We let $q(x)$ be the number of the sub-box that contains $x$ (provided that $x$ is indeed contained in one of them). The new value of $\hat{x}$ is then defined to be the center of this box. This can be described by a jump equation of the form

$$
\hat{x}\left(t_{i}^{+}\right)=g\left(i, q\left(x\left(t_{i}\right)\right), \hat{x}\left(t_{i}\right), \xi\left(t_{i}\right)\right) .
$$

We also update $\xi$ to reflect the fact that the size of the box known to contain $x$ was divided by $N$ as the result of the above procedure:

$$
\xi\left(t_{i}^{+}\right)=\frac{\xi\left(t_{i}\right)}{N} .
$$

${ }^{3}$ In [13] and [14], the total number of sub-boxes is $N$, i.e., $N^{1 / n}$ in each dimension. While our present choice of notation somewhat simplifies the formulas that follow, it is a trivial matter to adapt the results to the notation used in [13], [14]. Similarly, we could let $\xi$ be an $n$-vector instead of a scalar and thus allow more general rectilinear boxes, as done, e.g., in [6], [26]. 
Until the time $t_{i+1}$, we propagate $\xi$ according to some differential equation

$$
\dot{\xi}=g_{\xi}(t, \xi)
$$

and then the procedure is repeated. We can think of $\hat{x}$ and $\xi$ as being implemented synchronously on both ends of the communication channel, i.e., in the encoder and the decoder, starting from some known initial values. Having defined $\hat{x}$, we can now also close the loop using the control law (4).

It is convenient to rewrite the system dynamics in terms of the quantization-induced error $e:=\hat{x}-x$. Suppressing the equations for $\hat{x}$ and instead writing the equations for $e$ as before, we arrive at the closed-loop dynamics in the form

$$
\begin{aligned}
\dot{x} & =f(t, x, e) \quad \forall t \in\left[t_{i-1}, t_{i}\right] \\
\dot{e} & =g_{e}(t, x, e) \quad \forall t \in\left[t_{i-1}, t_{i}\right] \\
\dot{\xi} & =g_{\xi}(t, \xi) \quad \forall t \in\left[t_{i-1}, t_{i}\right] \\
e\left(t_{i}^{+}\right) & =h_{e}\left(i, x\left(t_{i}\right), e\left(t_{i}\right), \xi\left(t_{i}\right)\right) \\
\xi\left(t_{i}^{+}\right) & =h_{\xi}\left(\xi\left(t_{i}\right)\right)
\end{aligned}
$$

where $f$ is given by (18)

$$
g_{e}(t, x, e):=\tilde{f}(t, x+e, k(t, x+e))-\tilde{f}(t, x, k(t, x+e))
$$

and

$$
h_{e}(i, x, e, \xi):=g(i, q(x), x+e, \xi)-x .
$$

This is similar to (15)-(17) and becomes a special case of (5)-(7) if we define $z:=(e, \xi)$. Note that the equations for $e$ are not actually implemented, but only used for analysis. The above definition of $z$ allows us to fit QCS models (as well as NQCS models considered below) to the form (5)-(7), and consequently enables us to apply Definition 2 and Theorem 1 off-the-shelf. However, we will see in Remark 6 that sometimes one might want to treat $e$ and $\xi$ separately instead of combining them into a single vector.

To make sure that the quantizer is well defined, we need to assume that the initialization of $\xi$ and its evolution during continuous flows and jumps fulfills the following.

Assumption 1: A bound on the initial state $x(0)$ is known and $\xi$ and $\hat{x}$ are such that

$$
\|e(t)\|_{\infty} \leq \xi(t) \quad \forall t \geq 0 .
$$

This assumption basically means that the quantizer will never saturate. It is easy to enforce this assumption when the plant is linear; cf. [13]. In fact, since the quantized measurements are only taken at the times $t_{i}$, it is enough to require that (28) be satisfied at these times, and then we can set $g_{\xi}=0$.

Remark 1: QCS protocols considered in [1], [13] and elsewhere do not assume a known initial bound on the state, and instead implement an initial "zooming-out" stage whose goal is to obtain such a bound in some finite time. Since the size of the quantization box must grow during this zooming-out stage, the corresponding protocols are not UGES and so our analysis does not directly apply to them. However, the MATI bounds we provide in this paper remain accurate for these systems since, as shown in [1], [13], zooming out can be implemented with a very coarse quantizer or a very large MATI.

\section{NQCS With "Box" Protocols}

In this subsection, we combine quantization with time scheduling and show that the closed-loop system can be written in the form (5)-(7). As a special case, when there is only one node $(\ell=1)$, we obtain the previous QCS model. We note also that a slightly different class of quantization protocols with "zoom" studied in [1], [12], [15] can also be incorporated into our framework (see Section III-D below).

We again start with the plant (2) and the nominal control law (3). The next step is to implement this controller over a band-limited channel that will involve quantization and time scheduling. Namely, we apply the control law (4), where $\hat{x}$ is an estimate of $x$ generated as explained next. In between the transmission times, $\hat{x}$ is obtained by running a copy of the plant, given by (21), in the encoder and decoder. As in Section III-A, we partition the state vector as $x=\left(x_{1}, x_{2}, \ldots, x_{\ell}\right)$, where $\ell$ is the number of nodes and each $x_{j}$ has dimension $n_{j}$. The vector $\hat{x}$ is partitioned accordingly as $\hat{x}=\left(\hat{x}_{1}, \hat{x}_{2}, \ldots, \hat{x}_{\ell}\right)$, and the error vector

$$
e:=\hat{x}-x
$$

is partitioned as $e=\left(e_{1}, e_{2}, \ldots, e_{\ell}\right)$. We assume that for each $j \in\{1,2, \ldots, \ell\}$, we are given a quantizer $q_{j}$ with parameters $N \in \mathbb{N}, \hat{x}_{j} \in \mathbb{R}^{n_{j}}, \xi_{j} \in \mathbb{R}_{\geq 0}$ defined as explained in Section III-B. Here we take $N$ to be the same for all $j$, but this is not necessary (see also footnote 3 ).

We assume that at any transmission time $t_{i}$, a quantized value of only one $x_{j}, j \in\{1,2, \ldots, \ell\}$ will be transmitted over the network. To decide which component $x_{j}$ we are going to transmit, we use a scheduling function $s: \mathbb{N} \times \mathbb{R}^{n} \times \mathbb{R}^{n} \times \mathbb{R}^{\ell} \rightarrow\{1,2, \ldots, \ell\}$

$$
s=s(i, x, e, \xi) \text {. }
$$

Then, we quantize the component $x_{s\left(t_{i}\right)}\left(t_{i}\right)$ using the corresponding quantizer $q_{s\left(t_{i}\right)}$, and send the quantized value over the network to the decoder. To simplify the notation, we write the quantized value simply as $q\left(x_{s\left(t_{i}\right)}\left(t_{i}\right)\right)$ since it is always clear which quantizer is being used. The decoder takes the transmitted quantized value and resets the value of $\hat{x}_{s\left(t_{i}\right)}$ to be the center of the box corresponding to $q\left(x_{s\left(t_{i}\right)}\left(t_{i}\right)\right)$, while keeping all other components of $\hat{x}$ unchanged. In other words, at transmission times we have the equation shown at the bottom of the next page, which leads to

$$
e_{j}\left(t_{i}^{+}\right)= \begin{cases}h_{j}(i) & \text { if } j=s\left(t_{i}\right) \\ e_{j}\left(t_{i}\right) & \text { if } j \neq s\left(t_{i}\right)\end{cases}
$$

where $h_{j}(i)=h_{j}\left(i, x_{s\left(t_{i}\right)}\left(t_{i}\right), e_{s\left(t_{i}\right)}\left(t_{i}\right), \xi_{s\left(t_{i}\right)}\left(t_{i}\right)\right)$ and $h_{j}\left(i, x_{s}, e_{s}, \xi_{s}\right):=g_{j}\left(i, q\left(x_{s}\right), x_{s}+e_{s}, \xi_{s}\right)-x_{s}$. We find it convenient to rewrite (31) in the following form:

$$
\begin{aligned}
e\left(t_{i}^{+}\right) & =\left(I-\Psi\left(s\left(t_{i}\right)\right)\right) e\left(t_{i}\right)+\Psi\left(s\left(t_{i}\right)\right) H(i) \\
& =: h_{e}\left(i, x\left(t_{i}\right), e\left(t_{i}\right), \xi\left(t_{i}\right)\right)
\end{aligned}
$$


where $\Psi(s)$ was defined in (20), $H(i)=$ $H\left(i, x_{s\left(t_{i}\right)}\left(t_{i}\right), e_{s\left(t_{i}\right)}\left(t_{i}\right), \xi_{s\left(t_{i}\right)}\left(t_{i}\right)\right)$, and

$$
H\left(i, x_{s}, e_{s}, \xi_{s}\right):=\left(\begin{array}{c}
h_{1}\left(i, x_{s}, e_{s}, \xi_{s}\right) \\
\vdots \\
h_{\ell}\left(i, x_{s}, e_{s}, \xi_{s}\right)
\end{array}\right) .
$$

At each transmission time we need to adjust the size of the box $\left(\xi_{j}\right)$ that corresponds to the component $x_{j}$ that is being transmitted. In the sequel, we consider update laws of the following form:

$$
\xi\left(t_{i}^{+}\right)=h_{\xi}\left(i, s\left(t_{i}\right), \xi\left(t_{i}\right)\right)
$$

In particular, $\xi_{s\left(t_{i}\right)}$ is typically divided by $N$ as before. In between the transmission times, the vector $\xi=\left(\xi_{1}, \xi_{2}, \ldots, \xi_{\ell}\right)$ is propagated according to some differential equation of the form (24). To summarize, the closed-loop dynamics can be written as

$$
\begin{aligned}
\dot{x} & =f(t, x, e) \quad \forall t \in\left[t_{i-1}, t_{i}\right] \\
\dot{e} & =g_{e}(t, x, e) \quad \forall t \in\left[t_{i-1}, t_{i}\right] \\
\dot{\xi} & =g_{\xi}(t, \xi) \quad \forall t \in\left[t_{i-1}, t_{i}\right] \\
e\left(t_{i}^{+}\right) & =h_{e}\left(i, x\left(t_{i}\right), e\left(t_{i}\right), \xi\left(t_{i}\right)\right) \\
\xi\left(t_{i}^{+}\right) & =h_{\xi}\left(i, s\left(t_{i}\right), \xi\left(t_{i}\right)\right)
\end{aligned}
$$

where $f$ and $g_{e}$ are given by (18) and (27), and $s$ is given in (30). Note that we are again suppressing the dynamics of $\hat{x}$, which are superfluous in view of (29). Finally, it is clear that this system can be written in the form (5)-(7) by defining $z:=(e, \xi)$.

To make sure that the quantizer does not saturate and all the previous constructions are valid, we impose the following assumption (see also Remark 1 earlier).

Assumption 2: A bound on the initial state $x(0)$ is known and $\xi$ and $\hat{x}$ are such that

$$
\left\|e_{i}(t)\right\|_{\infty} \leq \xi_{i}(t) \quad \forall i \in\{1, \ldots, \ell\}, \forall t \geq 0 .
$$

More details on how to satisfy this assumption will be given later (when we specialize to linear plants in Section V).

\section{NQCS With "Zoom” Protocols}

In this section we consider another class of quantization protocols and combine them with time-scheduling protocols. These protocols were considered (for the case of quantization only) in [15]. That work in turn builds on the framework developed in [1], [12]; the models considered in these earlier papers differ only slightly from the ones we use in [15] and here. These quantization protocols are actually quite similar to the "box" protocols that we discussed in the previous subsections. The main difference is that typically, these zoom protocols do not strive to handle quantizers with as few quantization regions as possible, and consequently their implementation is somewhat more static; in particular, the center of the quantizer does not evolve according to a plant model between the transmission times as it does for "box" protocols. Some other differences between the two quantizer classes are purely notational, and these are reflected here to make a better link with the existing literature.

Assume that we are given $\ell$ quantizers $q_{j}: \mathbb{R}^{n_{j}} \rightarrow \mathcal{Q}_{j} \subset$ $\mathbb{R}^{n_{j}}, j \in\{1, \ldots, \ell\}$, where each $\mathcal{Q}_{j}$ is a finite (or countable) set of quantization points. Unlike in Section III-C, these quantizers do not have a moving center. Instead of describing them using the integer parameter $N$, we assume the following.

Assumption 3: There exist strictly positive numbers $M_{j}$ and $\Delta_{j}, j \in\{1, \ldots, \ell\}$ such that the following holds for all $j \in$ $\{1, \ldots, \ell\}$ and $z_{j} \in \mathbb{R}^{n_{j}}$ :

$$
\begin{aligned}
& \left|z_{j}\right| \leq M_{j} \Longrightarrow\left|q_{j}\left(z_{j}\right)-z_{j}\right| \leq \Delta_{j} \\
& \left|z_{j}\right|>M_{j} \Longrightarrow\left|q_{j}\left(z_{j}\right)\right|>M_{j}-\Delta_{j} .
\end{aligned}
$$

This formalism, proposed in [12], allows for quantization regions of general shapes, and incorporates as a special case rectilinear regions considered earlier in [1], [4]. Each quantizer $q_{j}$ also has a "zoom" parameter $\mu_{j}>0$, whose role is similar to that of $\xi_{j}$ from Section III-C

$$
q_{j}\left(x, \mu_{j}\right):=\mu_{j} q_{j}\left(\frac{x_{j}}{\mu_{j}}\right) .
$$

Writing $\mu=\left(\mu_{1}, \mu_{2}, \ldots, \mu_{\ell}\right)$, we define the overall quantizer as

$$
q(x, \mu)=\left(q_{1}(x, \mu), \ldots, q_{\ell}(x, \mu)\right) .
$$

Quantization is then combined with time scheduling in the same way as in Section III-C. At each $t_{i}$, the value of $\hat{x}_{s\left(t_{i}\right)}$ is reset to $q_{s\left(t_{i}\right)}\left(x_{s\left(t_{i}\right)}, \mu_{s\left(t_{i}\right)}\right)$. Here we let

$$
\dot{\hat{x}}=0
$$

between transmission times, which is actually more consistent with Section III-A. The vector of zoom parameters $\mu$ is also held constant between transmission times, while at transmission times we update it as follows:

$$
\mu\left(t_{i}^{+}\right)=\Omega_{\text {in }} \mu\left(t_{i}\right)
$$

where

$$
\Omega_{\text {in }}=\operatorname{diag}\left\{\Omega_{1}, \ldots, \Omega_{\ell}\right\}
$$

and $\Omega_{j} \in(0,1)$ for each $j$. Rewriting everything in $(x, e)$-coordinates as before, we obtain closed-loop dynamics of the form

$$
\begin{aligned}
\dot{x} & =f(t, x, e) \quad \forall t \in\left[t_{i-1}, t_{i}\right] \\
\dot{e} & =g_{e}(t, x, e) \quad \forall t \in\left[t_{i-1}, t_{i}\right] \\
\dot{\mu} & =0 \quad \forall t \in\left[t_{i-1}, t_{i}\right] \\
e\left(t_{i}^{+}\right) & =\left(I-\Psi\left(s\left(t_{i}\right)\right)\right) e\left(t_{i}\right) \\
& +\Psi\left(s\left(t_{i}\right)\right)\left(q\left(x\left(t_{i}\right), \mu\left(t_{i}\right)\right)-x\left(t_{i}\right)\right) \\
\mu\left(t_{i}^{+}\right) & =\Omega_{\text {in }} \mu\left(t_{i}\right)
\end{aligned}
$$

$$
\hat{x}_{j}\left(t_{i}^{+}\right)= \begin{cases}g_{j}\left(i, q\left(x_{s\left(t_{i}\right)}\left(t_{i}\right)\right), \hat{x}_{s\left(t_{i}\right)}\left(t_{i}\right), \xi_{s\left(t_{i}\right)}\left(t_{i}\right)\right) & \text { if } j=s\left(t_{i}\right) \\ \hat{x}_{j}\left(t_{i}\right) & \text { if } j \neq s\left(t_{i}\right)\end{cases}
$$


where $e$ is given by (29), $\Psi$ is given by (20), and the scheduling function $s$ has the same role as in the previous subsection and in general we have

$$
s=s(i, x, e, \mu) .
$$

To avoid quantizer saturation, we need the following.

Assumption 4: A bound on the initial state $x(0)$ is known and $\mu$ is such that for all $j \in\{1, \ldots, \ell\}$ we have

$$
\frac{\left|x_{j}(t)\right|}{\mu_{j}(t)} \leq M_{j} \quad \forall t \geq 0 .
$$

If $M_{j}=\infty$ for all $j \in\{1, \ldots, \ell\}$ (i.e., all quantizers have infinite range), then Assumption 4 always holds. For finite-range quantizers, this assumption plays a role similar to that of Assumption 2, and it is not difficult to enforce it at least for linear plants (see [12]). Remark 1 still applies here. We also note that in the presence of unknown external disturbances, we could not hope to satisfy Assumption 4 (or Assumption 2) from some time onward, and so more general quantization protocols would need to be employed; see [15] for more details.

\section{UGES PROTOCOLS}

In this section we consider various examples of protocols that may arise in Sections III-A, B, C, and D. More importantly, we show for each of these protocols that they are UGES in the sense of Definition 2. It will become apparent from our proofs that many other protocols, not considered here, can be treated in a similar manner. In Section V, the technical proofs presented in this section are combined with Theorem 1 to obtain a range of new results for emulation of classes of systems considered in Section III. This general approach is novel for any system that involves quantization with or without time scheduling.

\section{A. NCS Protocols}

In this subsection, we consider protocols of the form

$$
e^{+}=(I-\Psi(s)) e=h(i, e) \quad s=s(i, e)
$$

which arise in NCS considered in Section III-A; the function $\Psi$ is defined by (20). We typically assume that if a node $j$ transmits at time $t_{i}$, then $e_{j}$ is reset to zero at time $t_{i}^{+}$, i.e., $e_{j}\left(t_{i}^{+}\right)=0$. In other words, we ignore possible quantization effects in this subsection. However, we emphasize that this assumption is not needed in general and this will become clear in the sequel. We present two examples of protocols from [19], [27] and quote results from [19] that show that these protocols are UGES. Besides serving to illustrate the unifying nature of our results, the RR and TOD protocols that we present next are used in the sequel to generate several genuinely new protocols that combine quantization and time scheduling. Hence, results presented here are useful for our subsequent analysis.

1) Round Robin (RR) Protocol: The simplest time-scheduling protocol is round robin in which the node $j$ is transmitted periodically with period $\ell$, where $\ell$ is the total number of nodes. Such protocols are most widely studied in the literature (see, for instance, [8], [9], [11]) and the analysis we present was given in [19]. We specify now the protocol for this situation. First, we note that the scheduling function becomes in this case

$$
s=s(i)=j \text { if } i=j+k \ell \text { for some } k=0,1,2, \ldots \text {. }
$$

The following result was proved in [19]. We present the proof for completeness and because several proofs in the sequel rely on this computation.

Proposition 2: The RR protocol (39), (40) is UGES with the Lyapunov function $W(i, e):=\sqrt{\sum_{k=i}^{\infty}|\phi(k, i, e)|^{2}}$, where $\phi$ denotes the solution of the discrete-time system (39), (40) at time $k$ starting at time $i$ and initial condition $e$. In particular, we can take $a_{1}=1, a_{2}=\sqrt{\ell}$, and $\rho=\sqrt{\frac{\ell-1}{\ell}}$.

Proof of Proposition 2: Consider the Lyapunov function

$$
V(i, e)=\sum_{k=i}^{\infty}|\phi(k, i, e)|^{2}
$$

where it is obvious that $|e|^{2}=\phi^{2}(i, i, e) \leq V(i, e)$ for all $e$ and all $i \in \mathbb{N}$. Moreover, note that $\phi(k, i, e)=0$ for all $i \in \mathbb{N}$ and all $k \geq i+\ell$. Using this and also the fact that $|h(i, e)| \leq|e|$, we can write that

$$
V(i, e) \leq \ell|e|^{2}
$$

for all $e$ and all $i \in \mathbb{N}$. Furthermore, we have that

$$
V(i+1, h(i, e))=V(i, e)-|e|^{2} \leq \frac{\ell-1}{\ell} V(i, e)
$$

for all $e$ and all $i \in \mathbb{N}$, where (42) was used in the last step. Finally, with the definition $W(i, e):=\sqrt{V(i, e)}$ we obtain that (10) and (11) hold with $a_{1}=1, a_{2}=\sqrt{\ell}$, and $\rho=\sqrt{\frac{\ell-1}{\ell}}$, which completes the proof.

2) Try-Once-Discard (TOD) Protocol: In this section we consider the Try-Once-Discard (TOD) time-scheduling protocol proposed by Walsh et al. in [27], whose stability was analysed in [19]. In TOD protocol, the scheduling function takes the following form:

$$
s=s(e)=\min \left\{\arg \max _{i}\left|e_{i}\right|\right\} .
$$

In other words, we compare the norms of errors $e_{i}$ and then transmit the node $i$ that corresponds to the largest error. If several errors are the same, we transmit the node $i$ with a minimum index (priority to transmit in this case can be chosen differently). It was explained in [27] how TOD protocol can be implemented in Control Area Network (CAN); TOD cannot be directly implemented in many wired and any wireless networks. The following result was proved in [19].

Proposition 3: The TOD protocol (39), (43) is UGES with the Lyapunov function $W(e):=|e|$. In particular, we can take $a_{1}=a_{2}=1$ and $\rho=\sqrt{\frac{\ell-1}{\ell}}$.

Proof of Proposition 3: It is obvious that (10) holds with $a_{1}=a_{2}=1$ since $W(e)=|e|$. Consider arbitrary $e$ and suppose without loss of generality that $\left|e_{1}\right| \geq \max _{j \in[2, \ell]}\left|e_{j}\right|$, hence $s(e)=1$. Then, we can write

$$
\left|e_{1}\right|^{2}=\max _{j \in[1, \ell]}\left|e_{j}\right|^{2} \geq \frac{1}{\ell} \sum_{j=1}^{\ell}\left|e_{j}\right|^{2}=\frac{1}{\ell}|e|^{2} .
$$

Using the properties of TOD protocol, we can write

$$
\begin{aligned}
|(I-\Psi(e)) e|^{2} & =\sum_{j=1}^{\ell}\left|\left(1-\delta_{1 s(e)}\right) e_{j}\right|^{2}=\sum_{j=2}^{\ell}\left|e_{j}\right|^{2} \\
& =|e|^{2}-\left|e_{1}\right|^{2} .
\end{aligned}
$$


Finally, using (44) and (45) we have

$$
W\left(e^{+}\right) \leq \sqrt{|e|^{2}-\frac{1}{\ell}|e|^{2}}=\sqrt{\frac{\ell-1}{\ell}}|e|=\sqrt{\frac{\ell-1}{\ell}} W(e)
$$

which completes the proof.

\section{B. QCS Protocols}

We now prove that the quantization "box" protocol from Section III-B is UGES. As far as we are aware, this is the first analysis of stability properties for this protocol, taken separately from the continuous-time dynamics. However, protocols of this kind have been widely used in the literature as encoder/decoder models of communication channels between the plant and the controller; see, e.g., [6], [13], [14], [17], [21], [26] (although the focus in most of these works is somewhat different from the Lyapunov-based emulation design pursued here). The protocol is given by

$$
\begin{aligned}
e^{+} & =h_{e}(i, x, e, \xi) \\
\xi^{+} & =\frac{\xi}{N} .
\end{aligned}
$$

In view of Assumption 1 and other constructions in Section III-B, it is easy to show that there exists a $d_{1} \geq 0$ such that $h_{e}$ in (46) satisfies

$$
\left|h_{e}(i, x, e, \xi)\right| \leq d_{1}|\xi| \quad \forall i, x, e, \xi
$$

where we can take $d_{1}=\sqrt{n} / N$. The proof that we present below, although straightforward, is important since several other proofs in the sequel use similar constructions.

Proposition 4: Suppose that (47) holds. Then, the box quantization protocol (46) is UGES with the Lyapunov function $W(e, \xi):=\varepsilon|e|+|\xi|$, where $\varepsilon \in(0, \widetilde{\rho})$ and $\widetilde{\rho}=\frac{N-1}{d_{1} N}$. In particular, we can take $a_{1}=\min \{1, \varepsilon\}, a_{2}=1+\varepsilon$, and $\rho=\varepsilon d_{1}+1 / N$.

Proof of Proposition 4: To show UGES of the protocol (46) we first note that its model is a cascade of two systems. It is easy to see that the $\xi$-subsystem is UGES since with $U(\xi):=|\xi|$ we can write 4 .

$$
U\left(\xi^{+}\right)=\frac{1}{N}|\xi|=\frac{1}{N} U(\xi) .
$$

Moreover, the $e$-subsystem is ISS, uniformly in $x$, when $\xi$ is regarded as the input (and, in this case, one-step dead-beat stable when $\xi=0)$. Take $V(e):=|e|$ and use (47) to write

$$
V\left(e^{+}\right)=\left|h_{e}(i, x, e, \xi)\right| \leq d_{1}|\xi|=d_{1} U(\xi) .
$$

By defining $W(e, \xi):=\varepsilon V(e)+U(\xi)$ we have

$$
\min \{\varepsilon, 1\}|(e, \xi)| \leq W(e, \xi) \leq(1+\varepsilon)|(e, \xi)| .
$$

Finally, we can write

$$
\begin{aligned}
W\left(e^{+}, \xi^{+}\right) & =\varepsilon V\left(e^{+}\right)+U\left(\xi^{+}\right) \\
& \leq\left(\varepsilon d_{1}+\frac{1}{N}\right) U(\xi) \leq\left(\varepsilon d_{1}+\frac{1}{N}\right) W(e, \xi)
\end{aligned}
$$

${ }^{4}$ Note that $\xi(\cdot)$ in (24) is non-negative by definition and in principle we could use $U(\xi)=\xi$. However, in order to be consistent with our definition of UGES protocols, we need to show UGES of the auxiliary discrete-time system (46) which is defined on $\mathbb{R}^{n} \times \mathbb{R}$ and that is why we use $U(\xi):=|\xi|$. which completes the proof since our choice of $\varepsilon$ guarantees that $\rho=\varepsilon d_{1}+1 / N<1$.

\section{C. "Box” Protocols for NQCS}

The focus of this subsection are protocols of the form

$$
\begin{aligned}
e^{+} & =(I-\Psi(s)) e+\Psi(s) H\left(i, x_{s}, e_{s}, \xi_{s}\right) \\
& =h_{e}(i, x, e, \xi) \\
\xi^{+} & =h_{\xi}(i, s, \xi) \\
s & =s(i, x, e, \xi)
\end{aligned}
$$

that arise in Section III-C. The functions $H$ and $h_{\xi}$ depend on the quantization procedure, whereas the scheduling function $s$ depends on the time-scheduling procedure. Hence, the protocol (48) combines time scheduling and quantization. In view of (33) and Assumption 2, there exists a $d \geq 0$ such that

$$
|H(i, q(x), e, \xi)| \leq d|\xi| \quad \forall i, x, e, \xi .
$$

In what follows, this is all we need to know about the function $H$. Various combinations of scheduling with "box" protocols are possible and we consider two such protocols where we combine the RR and TOD time-scheduling protocols with the "box" quantization protocol. We are not aware of this class of protocols having been systematically studied in the literature.

1) RR Protocol With Quantization: In this section we combine the RR protocol considered in Subsection IV-A with the "box" quantization protocol considered in [13]. A somewhat similar (but more complicated) protocol was considered in [17]. The function $s$ in (48) is given by (40) and the function $\Psi$ is defined in (20). We know that $H$ satisfies (49). We let $h_{\xi}$ in (48) be given by

$$
h_{\xi}(i, s, \xi)=(I-\widetilde{\Psi}(s(i))) \xi+\frac{1}{N} \widetilde{\Psi}(s(i)) \xi
$$

where $N^{n_{s}}$ is the number of quantization levels for the corresponding quantizer $\left(q_{s}\right)$

$$
\widetilde{\Psi}(s):=\operatorname{diag}\left\{\delta_{1 s}, \ldots, \delta_{\ell s}\right\}
$$

(note that the matrices $\Psi$ and $\widetilde{\Psi}$ have different dimensions); $\delta_{i j}$ is the Kronecker symbol; $\ell$ denotes the number of nodes used in the time scheduling. This means that $\xi_{s(i)}$ is divided by $N$ while other components of $\xi$ remain unchanged. To summarize, the discrete-time system induced by the protocol is

$$
\begin{aligned}
e^{+} & =(I-\Psi(s(i))) e+\Psi(s(i)) H\left(i, x_{s(i)}, e_{s(i)}, \xi_{s(i)}\right) \\
\xi^{+} & =(I-\widetilde{\Psi}(s(i))) \xi+\frac{1}{N} \widetilde{\Psi}(s(i)) \xi=h_{\xi}(i, \xi) .
\end{aligned}
$$

Next, we show that the protocol (52) is Lyapunov UGES and in the proof we construct an appropriate Lyapunov function $W$ for this protocol.

Proposition 5: Suppose that (49) holds. Then, the protocol (52) is Lyapunov UGES. In particular, the inequalities (10) and (11) hold with $a_{1}=\min \{1, \varepsilon\}, a_{2}=\varepsilon \sqrt{\ell}+\sqrt{\frac{N^{2} \ell}{N^{2}-1}}$, and $\rho=\max \left\{\sqrt{\frac{\ell-1}{\ell}}, \varepsilon d \sqrt{\ell}+\widetilde{\rho}\right\}$, where $\varepsilon \in\left(0, \frac{1-\tilde{\rho}}{d \sqrt{\ell}}\right)$ and $\widetilde{\rho}=$ $\sqrt{\frac{N^{2} \ell-N^{2}+1}{N^{2} \ell}}$. 
Proof of Proposition 5: We construct the Lyapunov function for the protocol (52) in several steps. First, we construct a Lyapunov function for the $\xi$-subsystem. Then, we construct an ISS Lyapunov function for the $e$-subsystem when $\xi$ is regarded as an input. Finally, we combine the two functions (since the system is a cascade of two subsystems) into a Lyapunov function of the overall system. Thus the proof follows the pattern of the proof of Proposition 4, although the constructions and calculations are significantly more difficult.

Consider the $\xi$-subsystem in (52), and denote the solution of this system at time $k$ starting from $\xi$ at time $i$ as $\phi_{\xi}(k, i, \xi)$. Consider the function

$$
\widetilde{U}(i, \xi)=\sum_{k=i}^{\infty}\left|\phi_{\xi}(k, i, \xi)\right|^{2}
$$

where it is obvious that $|\xi|^{2}=\phi_{\xi}^{2}(i, i, \xi) \leq \widetilde{U}(i, \xi)$ for all $\xi \in \mathbb{R}^{\ell}, i \in \mathbb{N}$. Moreover, note that for arbitrary $i \in \mathbb{N}$ and all $k \in[i+j \ell, i+(j+1) \ell-1], j=0,1, \ldots$ we have that

$$
\left|\phi_{\xi}(k, i, \xi)\right|^{2} \leq\left(\frac{1}{N^{2}}\right)^{j}|\xi|^{2}
$$

and hence we can write for all $i$ and $\xi$ that

$$
\widetilde{U}(i, \xi) \leq \ell \sum_{j=0}^{\infty}\left(\frac{1}{N^{2}}\right)^{j}|\xi|^{2}=\frac{\ell}{1-\frac{1}{N^{2}}}|\xi|^{2}=\frac{N^{2} \ell}{N^{2}-1}|\xi|^{2} .
$$

Furthermore, we have that

$$
\begin{aligned}
\widetilde{U}\left(i+1, h_{\xi}(i, \xi)\right) & =\widetilde{U}(i, \xi)-|\xi|^{2} \\
& \leq\left(1-\frac{N^{2}-1}{N^{2} \ell}\right) \widetilde{U}(i, \xi) \\
& =\frac{N^{2} \ell-N^{2}+1}{N^{2} \ell} \widetilde{U}(i, \xi)
\end{aligned}
$$

for all $\xi \in \mathbb{R}^{\ell}$ and all $i \in \mathbb{N}$. Defining $U(i, \xi):=\sqrt{\widetilde{U}(i, \xi)}$, we have for all $(i, \xi)$ that

$$
\begin{aligned}
|\xi| & \leq U(i, \xi) \leq \tilde{a}|\xi| \\
U\left(i+1, h_{\xi}(i, \xi)\right) & \leq \tilde{\rho} U(i, \xi)
\end{aligned}
$$

where $\tilde{a}=\sqrt{\frac{N^{2} \ell}{N^{2}-1}}, \tilde{\rho}:=\sqrt{\frac{N^{2} \ell-N^{2}+1}{N^{2} \ell}}$. Note that $0<\widetilde{\rho}<1$ because $N>1$ and $l \geq 1$.

Next, we find an ISS Lyapunov function for the $e$-subsystem in (52) when $\xi$ is regarded as an input. To do this we first consider the system

$$
e^{+}=(I-\Psi(s(i))) e=: \widetilde{h}(i, e) .
$$

A Lyapunov function $W$ for this system is given in Proposition 2. We relabel this function as $V(i, e)$. Then, for all $i$ and $e$ we have

$$
\begin{aligned}
|e| & \leq V(i, e) \leq \sqrt{\ell}|e| \\
V(i+1, \widetilde{h}(i, e)) & \leq \sqrt{\frac{\ell-1}{\ell}} V(i, e) .
\end{aligned}
$$

Moreover, $V$ is globally Lipschitz with the Lipschitz constant equal to $\sqrt{\ell}$ (see Lemma 2 in Appendix). We show that the same function is an ISS Lyapunov function for the $e$-subsystem in (52):

$V\left(i+1, h_{e}(i, x, e, \xi)\right)=V(i+1, \widetilde{h}(i, e))+$

$$
\begin{aligned}
& V\left(i+1, h_{e}(i, x, e, \xi)\right)-V(i+1, \widetilde{h}(i, e)) \leq \sqrt{\frac{\ell-1}{\ell}} V(i, e) \\
& +\left|V\left(i+1, h_{e}(i, x, e, \xi)\right)-V(i+1, \widetilde{h}(i, e))\right| \\
& \leq \sqrt{\frac{\ell-1}{\ell}} V(i, e)+\sqrt{\ell}\left|h_{e}(i, x, e, \xi)-\widetilde{h}(i, e)\right| \\
& =\sqrt{\frac{\ell-1}{\ell}} V(i, e)+\sqrt{\ell}\left|\Psi(s(i)) H\left(i, x_{s(i)}, e_{s(i)}, \xi_{s(i)}\right)\right| \\
& \leq \sqrt{\frac{\ell-1}{\ell}} V(i, e)+d \sqrt{\ell} U(i, \xi)
\end{aligned}
$$

where we used the global Lipschitz property of $V$, (49), and (54). Finally, we let

$$
W(i, e, \xi):=\varepsilon V(i, e)+U(i, \xi)
$$

where $\varepsilon$ is strictly positive. We can write for all $i, e, \xi$ that $\min \{1, \varepsilon\}|(e, \xi)| \leq W(i, e, \xi) \leq\left(\varepsilon \sqrt{\ell}+\sqrt{\frac{N^{2} \ell}{N^{2}-1}}\right)|(e, \xi)|$.

Thus (10) holds since $\varepsilon>0, \ell \geq 1$ and $N>1$. Moreover, denoting $W^{+}:=W\left(i+1, h_{e}(i, x, e, \xi), h_{\xi}(i, \xi)\right)$, we show that (11) holds by using (55) and (56) as follows:

$$
\begin{aligned}
W^{+} & =\varepsilon V\left(i+1, h_{e}(i, x, e, \xi)\right)+U\left(i+1, h_{\xi}(i, \xi)\right) \\
& \leq \varepsilon \sqrt{\frac{\ell-1}{\ell}} V(i, e)+(\varepsilon d \sqrt{\ell}+\widetilde{\rho}) U(i, \xi) \\
& \leq \max \left\{\sqrt{\frac{\ell-1}{\ell}}, \varepsilon d \sqrt{\ell}+\widetilde{\rho}\right\}(\varepsilon V(i, e)+U(i, \xi)) \\
& =\rho W(i, e, \xi)
\end{aligned}
$$

where $\rho:=\max \left\{\sqrt{\frac{\ell-1}{\ell}}, \varepsilon d \sqrt{\ell}+\widetilde{\rho}\right\}$ and $\widetilde{\rho}$ was defined earlier. Note that $\rho<1$ since $\varepsilon<\frac{1-\widetilde{\rho}}{d \sqrt{\ell}}$.

2) TOD Protocol With Quantization: In this subsection we consider a combination of TOD protocol and quantization. We believe that this protocol has not been considered previously in the literature. The protocol is given by

$$
\begin{aligned}
e^{+} & =(I-\Psi(s)) e+\Psi(s) H\left(i, x_{s}, e_{s}, \xi_{s}\right)=h_{e}(i, x, e, \xi) \\
\xi^{+} & =(I-\widetilde{\Psi}(s)) M(s(e), \xi)+\frac{1}{N} \widetilde{\Psi}(s) \xi=h_{\xi}(e, \xi)
\end{aligned}
$$

where $\Psi$ is defined in (20), $\widetilde{\Psi}(s)$ is defined in (51), $s$ is defined in (43), $H$ satisfies (49), and $M(s, \xi)=\left(m_{1}(s, \xi), \ldots, m_{\ell}(s, \xi)\right)$, where $m_{j}(s, \xi):=\min \left\{\xi_{s}, \xi_{j}\right\}$. In other words, the updated value of $\xi_{j}$ for any $j \in\{1, \ldots, \ell\}$ satisfies the following:

$$
\xi_{j}^{+}= \begin{cases}\frac{\xi_{j}}{N} & \text { if } j=s \\ \xi_{s} & \text { if } \xi_{j} \geq \xi_{s} \text { and } j \neq s \\ \xi_{j} & \text { if } \xi_{j} \leq \xi_{s} \text { and } j \neq s .\end{cases}
$$

The above protocol first compares the errors $e_{i}$ in individual nodes and then transmits a quantized version of the measurement in the node with the largest error. Note that the $\xi_{j}$ corresponding to this node is divided by $N$ after the transmission. Moreover, since we already know which node has the largest error, any of the $\xi_{i}$ 's corresponding to other nodes that are larger than $\xi_{j}$ are reset to be equal to $\xi_{j}$ (recall Assumption 2). In this manner the quantization protocol is working more efficiently. 
Remark 2: While not immediately obvious, the protocol (57), (58) is quite a natural way to combine TOD with a box quantization protocol. We next comment on a few alternative related protocols. First, note that a naive update for $\xi$ as follows:

$$
\xi^{+}=(I-\widetilde{\Psi}(s)) \xi+\frac{1}{N} \widetilde{\Psi}(s) \xi
$$

where $s$ is defined in (43) may not work and, in particular, we were unable to prove that this modified protocol is Lyapunov UGES.

On the other hand, consider the following modification of the box protocol for NQCS given by (57):

$$
\begin{aligned}
e^{+} & =(I-\Psi(s)) e+\Psi(s) H\left(i, e_{s}, \xi_{s}\right) \\
\xi^{+} & =(I-\widetilde{\Psi}(s)) \xi+\frac{1}{N} \widetilde{\Psi}(s) \xi \\
s & =\min \left\{\arg \max _{i} \xi_{i}\right\}
\end{aligned}
$$

which was considered in [6] (see also [16]). The functions $\Psi$, $\widetilde{\Psi}$ and $H$ are the same as in (57). Note that this protocol applies the TOD time scheduling based on the values of $\xi_{i}$ instead of the values of $\left|e_{i}\right|$ that were used in (57).

We note that this protocol is not UGES in the sense of our Definition 2. Indeed, consider the following initial condition:

$$
e^{*}=\left(0, e_{2}, \ldots, e_{\ell}\right) ; \quad \xi^{*}=\left(\xi_{1}, 0,0, \ldots, 0\right)
$$

where $\xi_{1}>0$ and there exists $j \in\{2,3, \ldots, \ell\}$ such that $e_{j} \neq 0$. Denote the $e$ and $\xi$ parts of the solution of the system (59)-(61) initialized at $\left(e^{*}, \xi^{*}\right)$ as $\phi_{e}\left(k, e^{*}, \xi^{*}\right)$ and $\phi_{\xi}\left(k, e^{*}, \xi^{*}\right)$, respectively. Since $\xi_{1}>0$ and $\xi_{j}=0$, $\forall j=2,3, \ldots, \ell$ we have that for all time $s \equiv 1$ (node 1 will always transmit) and as a consequence we have for all $k \in \mathbb{N}$ :

$\phi_{e}\left(k, e^{*}, \xi^{*}\right)=e^{*} \neq 0 ; \quad \phi_{\xi}\left(k, e^{*}, \xi^{*}\right)=\left(\frac{\xi_{1}}{N^{k}}, 0,0, \ldots, 0\right)$.

Since $\phi_{e}$ does not converge to zero, the system is not UGES in the sense of Definition 2. Nevertheless, stability of linear NQCS with this protocol was proved in [6] under appropriate conditions. Hence, our definition of UGES protocols is too strong in this case and Theorem 1 cannot be applied directly. We will discuss in Section $\mathrm{V}$ how to modify our results to deal with this kind of situation (see Remark 6).

We prove next that the protocol (57) is UGES.

Proposition 6: Suppose that (49) holds. Then, the protocol (57) is Lyapunov UGES. In particular, the inequalities (10) and (11) hold with $a_{1}=\min \{1, \varepsilon\}, a_{2}=1+\varepsilon$, and $\rho=\max \left\{\sqrt{\frac{\ell-1}{\ell}}, \varepsilon d+\widetilde{\rho}\right\}$, where $\varepsilon \in\left(0, \frac{1-\tilde{\rho}}{d}\right)$, $\tilde{\rho}=\max \left\{\sqrt{\frac{\ell+\alpha^{2}-1}{\ell}}, \sqrt{\frac{N^{2} \ell-\alpha^{2} N^{2}+\alpha^{2}}{N^{2} \ell}}\right\}$, and $\alpha \in(0,1)$ is arbitrary.

Proof of Proposition 6: We construct a Lyapunov function for the system (57) in several steps. First, we show that the $\xi$-subsystem is UGES, uniformly in $e$ (or in other words, exponentially ISS with zero gain when $e$ is considered as an input to the system). Let the Lyapunov function candidate be $U(\xi):=|\xi|$. In the sequel we often use the following fact:

$$
\frac{1}{\ell}|\xi|^{2} \leq \max _{i} \xi_{i}^{2}
$$

Let $\alpha \in(0,1)$ be arbitrary. Let $j \in\{1, \ldots, \ell\}$ be such that $\xi_{j}=\max _{i} \xi_{i}$ and consider two cases.

CASE 1: $\xi_{s} \leq \alpha \max _{i} \xi_{i}=\alpha \xi_{j}$. Using (58), (63) we have

$$
\begin{aligned}
& U^{2}\left(h_{\xi}(e, \xi)\right)=\left(\xi_{j}^{+}\right)^{2}+\sum_{k \neq j}\left(\xi_{k}^{+}\right)^{2} \leq \xi_{s}^{2}+\sum_{k \neq j} \xi_{k}^{2} \\
& \leq \alpha^{2} \xi_{j}^{2}+\sum_{k \neq j} \xi_{k}^{2}=|\xi|^{2}-\left(1-\alpha^{2}\right) \max _{i} \xi_{i}^{2} \\
& \quad \leq|\xi|^{2}-\frac{1-\alpha^{2}}{\ell}|\xi|^{2} \leq \frac{\ell+\alpha^{2}-1}{\ell} U^{2}(\xi)=: \rho_{1}^{2} U^{2}(\xi) .
\end{aligned}
$$

Note that $\rho_{1}<1$ for all $\ell \geq 1$.

CASE 2: $\xi_{s}>\alpha \max _{i} \xi_{i}=\alpha \xi_{j}$. Using (58), (63) we have

$$
\begin{aligned}
& U^{2}\left(h_{\xi}(e, \xi)\right)=\sum_{k=1}^{\ell}\left(\xi_{k}^{+}\right)^{2}=\sum_{k \neq s}\left(\xi_{k}^{+}\right)^{2}+\frac{1}{N^{2}} \xi_{s}^{2} \\
& \leq \sum_{k \neq s} \xi_{k}^{2}+\frac{1}{N^{2}} \xi_{s}^{2}=\sum_{k=1}^{\ell} \xi_{k}^{2}-\left(1-\frac{1}{N^{2}}\right) \xi_{s}^{2} \\
& \leq|\xi|^{2}-\left(1-\frac{1}{N^{2}}\right) \alpha^{2} \max _{i} \xi_{i}^{2} \\
& \leq|\xi|^{2}-\left(1-\frac{1}{N^{2}}\right) \alpha^{2} \frac{1}{\ell}|\xi|^{2} \\
& =\frac{N^{2} \ell-\alpha^{2} N^{2}+\alpha^{2}}{N^{2} \ell} U^{2}(\xi)=: \rho_{2}^{2} U^{2}(\xi) .
\end{aligned}
$$

Note that $\rho_{2}<1$ whenever $N>1$.

By defining $\widetilde{\rho}:=\max \left\{\rho_{1}, \rho_{2}\right\}$, we can write that for all $\xi, e$ we have

$$
U\left(h_{\xi}(e, \xi)\right) \leq \widetilde{\rho} U(\xi) .
$$

Next, we look at $e$ by defining $V(e)=|e|$. We showed in Proposition 3 that the following holds:

$$
V((I-\Psi(e)) e) \leq \sqrt{\frac{\ell-1}{\ell}} V(e) .
$$

Hence, using (49), (57) and the triangle inequality we can write

$$
\begin{aligned}
& V\left(h_{e}(i, x, e, \xi)\right)=\left|(I-\Psi(e)) e+\Psi(e) H\left(i, x_{s}, e_{s}, \xi_{s}\right)\right| \\
& \quad \leq V((I-\Psi(e)) e)+\left|\Psi(e) H\left(i, x_{s}, e_{s}, \xi_{s}\right)\right| \\
& \quad \leq \sqrt{\frac{\ell-1}{\ell}} V(e)+d U(\xi) .
\end{aligned}
$$

We have proved that the $e$-subsystem is ISS with the ISS Lyapunov function $V(e)$. We combine the two Lyapunov functions $U$ and $V$ to get an overall Lyapunov function in the form $W(\xi, e)=\varepsilon V(e)+U(\xi)$. The remaining steps of the proof are the same as in the proof of Proposition 5.

\section{D. "Zoom" Protocols for NQCS}

The focus of this subsection are protocols of the form

$$
\begin{aligned}
e^{+} & =(I-\Psi(s)) e+\Psi(s)(q(x, \mu)-x)=: h_{e}(i, x, e, \mu) \\
\mu^{+} & =\Omega_{\text {in }} \mu=: h_{\mu}(\mu) \\
s & =s(i, x, e, \mu)
\end{aligned}
$$

that arise in Section III-D. The protocol (64) combines time scheduling and quantization in a different manner from the protocols considered in the previous subsection. We are not aware of this class of protocols having been previously considered in 
the literature. In the special case when $\ell=1$ (no time scheduling) these protocols were considered in [15]. We will combine $\mathrm{RR}$ and TOD protocols with the zooming quantization protocol and show that both of these combined protocols are UGES. A direct consequence of these results is that the zooming-in protocols considered in [15] are UGES.

1) RR With Zoom Protocols: In this section, we consider the protocol (64) with $\Psi$ defined by (20) and $s$ defined by (40). In this case, we can prove:

Proposition 7: Suppose that Assumptions 3 and 4 hold and $\max _{j} \Omega_{j}<1$. Then, the protocol (64) with (40) is Lyapunov UGES. In particular, the inequalities (10) and (11) hold with

$$
\begin{gathered}
\varepsilon \in\left(0, \frac{1-\max _{j} \Omega_{j}}{\sqrt{\ell} \max _{j} \Delta_{j}}\right), a_{1}=\min \{1, \varepsilon\}, \quad a_{2}=1+\varepsilon \sqrt{\ell} \\
\rho=\max \left\{\sqrt{\frac{\ell-1}{\ell}}, \varepsilon \sqrt{\ell} \max _{j} \Delta_{j}+\max _{j} \Omega_{j}\right\} .
\end{gathered}
$$

Proof of Proposition 7: The system is a cascade of an ISS system and a UGES system, and the proof proceeds similarly to that of Proposition 5. Let the function (41) be denoted as $V$. We show that $\widetilde{V}(i, e):=\sqrt{V(i, e)}$ is the ISS Lyapunov function for the $e$-subsystem. Note first that we have

$$
|e| \leq \widetilde{V}(i, e) \leq \sqrt{\ell}|e| .
$$

Moreover, since $\widetilde{V}$ is globally Lipschitz with Lipschitz constant equal to $\sqrt{\ell}$ (see Lemma 2 in Appendix), we obtain

$$
\widetilde{V}\left(i+1, e^{+}\right)=\sqrt{\frac{\ell-1}{\ell}} \widetilde{V}+\sqrt{\ell} \max _{j} \Delta_{j}|\mu| .
$$

It is also easy to see that the function $U(\mu):=|\mu|$ satisfies

$$
U\left(\mu^{+}\right) \leq \max _{j} \Omega_{j}|\mu|=\max _{j} \Omega_{j} U(\mu) .
$$

We define $W(i, x, e)=\varepsilon V(i, e)+U(\mu)$. Then we have

$$
\min \{1, \varepsilon\}|(e, \mu)| \leq W(i, e, \mu) \leq(1+\varepsilon \sqrt{\ell})|(e, \mu)| .
$$

Finally, we have

$$
\begin{aligned}
& W\left(i+1, e^{+}, \mu^{+}\right) \leq \varepsilon \widetilde{V}\left(i+1, e^{+}\right)+U\left(\mu^{+}\right) \\
& \leq \varepsilon \sqrt{\frac{\ell-1}{\ell}} \widetilde{V}(i, e)+\varepsilon \sqrt{\ell} \max _{j} \Delta_{j} U(\mu)+\max _{j} \Omega_{j} U(\mu) \\
& \leq \max \left\{\sqrt{\frac{\ell-1}{\ell}}, \varepsilon \sqrt{\ell} \max _{j} \Delta_{j}+\max _{j} \Omega_{j}\right\} \times(\varepsilon \widetilde{V}(i, e)+U(\mu)) \\
& =\rho W(i, e, \mu)
\end{aligned}
$$

and $\rho<1$ because $\varepsilon<\frac{1-\max _{j} \Omega_{j}}{\sqrt{\ell} \max _{j} \Delta_{j}}$.

2) TOD With Zoom Protocols: In this case, we consider (64) where $\Psi$ is defined in (20) and $s$ is defined in (43). We can prove the following:

Proposition 8: Suppose that Assumptions 3 and 4 hold and $\max _{j} \Omega_{j}<1$. Then, the protocol (64) with (43) is UGES with the Lyapunov function $W(e, \mu):=\varepsilon|e|+|\mu|$. In particular, the inequalities (10) and (11) hold with

$$
\begin{aligned}
& \varepsilon \in\left(0, \frac{1-\max _{j} \Omega_{j}}{\max _{j} \Delta_{j}}\right), a_{1}=\min \{1, \varepsilon\}, \quad a_{2}=1+\varepsilon, \\
& \rho=\max \left\{\sqrt{\frac{\ell-1}{\ell}}, \varepsilon \max _{j} \Delta_{j}+\max _{j} \Omega_{j}\right\} .
\end{aligned}
$$

Proof of Proposition 8: The system is again a cascade connection of an ISS and a UGES system. Indeed, it is easy to see that $V(e)=|e|$ is an ISS Lyapunov function for the $e$-subsystem:

$$
\begin{aligned}
V\left(h_{e}(x, e, \mu)\right) & =|(I-\Psi(e)) e+\Psi(e)(q(x, \mu)-x)| \\
& \leq|(I-\Psi(e)) e|+|\Psi(e)(q(x, \mu)-x)| \\
& \leq \sqrt{\frac{\ell-1}{\ell}} V(e)+\left(\max _{j} \Delta_{j}\right)|\mu| .
\end{aligned}
$$

Moreover, using $U(\mu):=|\mu|$ we can show UGES of the $\mu$-subsystem

$$
U\left(h_{\mu}(\mu)\right)=\left|\Omega_{\mathrm{in}} \mu\right| \leq\left(\max _{j} \Omega_{j}\right) U(\mu) .
$$

Combining these two Lyapunov functions as before gives us the Lyapunov function for the overall protocol in the form $W(e, \mu)=\varepsilon V(e)+U(\mu)$.

Remark 3: Note that when $\ell=1$ (one node only), we have in particular that $\Psi(e)=I$ and therefore

$$
\begin{aligned}
e^{+} & =q(x, \mu)-x=h_{e}(x, e, \mu) \\
\mu^{+} & =\Omega_{\text {in }} \mu=h_{\mu}(\mu)
\end{aligned}
$$

and we get for the ISS Lyapunov function $V$

$$
V\left(h_{e}(x, e, \mu)\right) \leq 0 \cdot V(e)+\left(\max _{j} \Delta_{j}\right)|\mu| .
$$

Everything else stays the same. This is exactly the situation considered in [15] and, hence, we can conclude that the zoom protocols considered in [15] are UGES.

\section{MAIN RESUlts}

In this section we demonstrate the utility and generality of our unifying approach to the controller emulation design for systems considered in Section III. In particular, we state a range of corollaries that provide MATI bounds guaranteeing stability for linear plants with linear emulated controllers and various quantization and/or time-scheduling UGES protocols considered in Section IV. This makes the formulas for MATI more concrete but we emphasize that the same approach can be applied to nonlinear plants with nonlinear (and dynamic) controllers. We thus consider the plant dynamics

$$
\dot{x}=A x+B u .
$$

The first step in the emulation approach is to design a linear controller

$$
u=K x
$$


so that the closed-loop system (65), (66) is exponentially stable, i.e., the matrix $A+B K$ is Hurwitz. Note that at the controller design stage the network is ignored but the same controller is implemented over a band-limited channel that will involve quantization and/or time scheduling. In particular, we apply a linear controller

$$
u=K \hat{x}
$$

where $\hat{x}$ can be thought of as the estimate of $x$ and it can be obtained in different ways depending on the type of the network over which controller is implemented.

The second step in the emulation approach is to select an appropriate UGES protocol, such as the ones analyzed in Section IV, and implement the controller over an appropriate network with sufficiently small MATI. Hence, MATI is a design parameter and its upper bound that guarantees stability of the closed loop can be obtained by applying Theorem 1 . To this end we will first show that the closed-loop model has the form (5)-(7). Indeed, using (29) we rewrite the closed-loop system (65), (67) as follows:

$$
\dot{x}=(A+B K) x+B K e .
$$

Note that (68) is a special case of the (5) and this will be the same in each of the subsections that follow. However, (6) and (7) will take different forms in the subsequent subsections depending on the properties of the network and its protocol as specified in Section III and below.

In order to avoid repetitions in the proofs that follow, we outline the main steps common to all of them. It is clear that since $A+B K$ is Hurwitz, the system (68) is ISS from $e$ to $x$ with a linear gain ${ }^{5} \kappa(s)=\gamma \cdot s, \gamma \geq 0$ and a class exp- $\mathcal{K} \mathcal{L}$ function $\beta$. Hence, item i) of Theorem 1 is satisfied. As explained above, we will assume that the network protocol is selected to be UGES and hence item ii) of Theorem 1 will also hold automatically in all the results that follow. Note that the Lyapunov functions will be different for different protocols and their derivations can be found in Section IV. The last thing to check is that item iii) of Theorem 1 holds for particular protocols that we will consider below and, finally, we would need to select MATI that is sufficiently small by applying the (13) in the item iv) of Theorem 1 . Note that by using this approach we will be able to state many results on emulation for NCS, QCS as well as NQCS with various UGES protocols.

\section{A. Emulation of NCS}

In this subsection we present two results for NCS systems that consist of linear plants with linear state feedback controllers with the full state being sent over the network. Moreover, we consider two cases of network protocols: RR and TOD protocols considered in Section IV-A. While these results follow directly from [19], it is instructive to state them in order to illustrate the unifying nature of our results. Moreover, this particular situation was not considered in [19] although we believe it is interesting in its own right and worth reporting.

\footnotetext{
${ }^{5}$ This gain can be estimated from standard ISS Lyapunov inequalities with quadratic Lyapunov functions.
}

Under the zero order hold update for $\hat{x}$ as in Section III, using (15)-(18) and (68), we can write the evolution of $e$ between transmission instants and its updates at jumps as follows:

$$
\begin{aligned}
\dot{e} & =-B K e-(A+B K) x \quad \forall t \in\left[t_{i-1}, t_{i}\right] \\
e\left(t_{i}^{+}\right) & =h\left(i, e\left(t_{i}\right)\right) .
\end{aligned}
$$

Hence, the closed-loop NCS with emulated controller implemented over network is given by (68), (69), (70) and (1), where the protocol $h(i, e)$ can be any time-scheduling protocol, such as the RR and TOD protocols considered in Section IV-A. Next we present two results that provide the bound on MATI for which this systems is exponentially stable in the case when the protocol is RR or TOD.

\section{1) RR Protocol:}

Corollary 9: Consider the NCS (68)-(70) and (1) with the RR protocol (39), (40) that has $\ell$ nodes. Suppose that $K$ is designed so that $A+B K$ is Hurwitz and let $\kappa(s)=\gamma \cdot s, \gamma \geq 0$ be the linear ISS gain for the system (68) from $e$ to $x$. Then, the NCS is UGES if MATI satisfies $\tau \in\left(0, \tau^{*}\right)$, where

$$
\tau^{*}:=\frac{1}{\sqrt{\ell}\|B K\|} \ln \left(\frac{\sqrt{\ell}\|B K\|+\gamma \sqrt{\ell}\|A+B K\|}{\sqrt{\ell-1}\|B K\|+\gamma \sqrt{\ell}\|A+B K\|}\right) .
$$

Proof of Corollary 9: As already outlined, we need to show that with $W$ from Proposition 2 item iii) of Theorem 1 holds, and we need to calculate the bound on MATI using item iv) of the theorem. First, note that we can show that for all $i$ and almost all $e$ we have that the following holds (see Lemma 2 in Appendix): $|\partial W(i, e) / \partial e| \leq \sqrt{\ell}$. Using this and the fact that $|e| \leq W(i, e)$ (see Proposition 2) we can write:

$$
\begin{aligned}
& \left\langle\frac{\partial W(i, e)}{\partial e},-B K e-(A+B K) x\right\rangle \\
& \leq\left|\frac{\partial W(i, e)}{\partial e}\right|(\|B K\||| e|+\|A+B K\|||x|) \\
& \leq \sqrt{\ell}\|B K\| W(i, e)+\sqrt{\ell}\|A+B K\||x| .
\end{aligned}
$$

Hence, item iii) of Theorem 1 holds with $L=\sqrt{\ell}\|B K\|$ and $c=\sqrt{\ell}\|A+B K\|$. Moreover, from Proposition 2 we have that $\rho=\sqrt{\frac{\ell-1}{\ell}}$. A direct substitution of these numbers into (13) gives (71).

\section{2) TOD Protocol:}

Corollary 10: Consider the NCS (68)-(70) and (1) with the TOD protocol (39), (43) that has $\ell$ nodes. Suppose that $K$ is designed so that $A+B K$ is Hurwitz and let $\kappa(s)=\gamma \cdot s, \gamma \geq 0$ be the linear ISS gain for the system (68) from $e$ to $x$. Then, the NCS is UGES if MATI satisfies $\tau \in\left(0, \tau^{*}\right)$, where

$$
\tau^{*}:=\frac{1}{\|B K\|} \ln \left(\frac{\sqrt{\ell}\|B K\|+\gamma \sqrt{\ell}\|A+B K\|}{\sqrt{\ell-1}\|B K\|+\gamma \sqrt{\ell}\|A+B K\|}\right) .
$$

Proof of Corollary 10: The proof follows almost the same steps as the proof of Corollary 9 except that we now use the Lyapunov function $W(e)=|e|$ that was used in Proposition 3 to show UGES of TOD protocol. Note that in this case we have 
for almost all $e$ that $|\partial W(e) / \partial e| \leq 1$. The rest of the proof follows the same steps as in Corollary 9 and is omitted.

Remark 4: Note that $\tau^{*}$ for RR protocol given by (71) is smaller than $\tau^{*}$ for TOD protocol given by (72) whenever $\ell>1$ and they are equal when $\ell=1$. Hence, one may be tempted to conclude that TOD protocol will perform better for larger values of MATI than the RR protocol. Unfortunately, this cannot be concluded from the above inequalities since our analysis is Lyapunov based and, hence, another choice of Lyapunov functions for the two protocols will lead to different expressions for $\tau^{*}$. Nevertheless, it was observed in some examples [19], [27] that the TOD protocol stabilized the system for larger MATI than the $\mathrm{RR}$ protocol.

\section{B. Emulation of $Q C S$}

In this subsection, we present results on emulation for QCS given in Section III-B and protocols given in Section IV-B. In this case, we will be running a copy of the plant in the encoder and decoder

$$
\dot{\hat{x}}=A \hat{x}+B u
$$

and, hence, the error $e=\hat{x}-x$ satisfies

$$
\dot{e}=A e \quad \forall t \in\left[t_{i-1}, t_{i}\right]
$$

between the quantization updates. The evolution of $\xi$ between the quantization updates is given by

$$
\dot{\xi}=\|A\|_{\infty} \xi \quad \forall t \in\left[t_{i-1}, t_{i}\right] .
$$

While versions of the following result have already appeared in the literature, the proof given below is original as it uses Theorem 1 and the novel conclusion in Proposition 4.

Corollary 11: Consider the QCS (68), (74), (75) and (1) with the box protocol (46). Suppose that $K$ is designed so that $A+$ $B K$ is Hurwitz and let $\kappa(s)=\gamma \cdot s, \gamma \geq 0$ be the ISS gain for the system (68) from $e$ to $x$. Then, the QCS is UGES if MATI satisfies $\tau \in\left(0, \tau^{*}\right)$, where:

$$
\tau^{*}:=\frac{1}{\sqrt{n}\|A\|_{\infty}} \ln \left(\frac{N}{\varepsilon d_{1} N+1}\right)
$$

where $N>1$ comes from (46), $n$ is the dimension of $x, d_{1}=$ $\sqrt{n} / N$ and $\varepsilon \in\left(0, \frac{N-1}{N d}\right)$.

Proof of Corollary 11: The proof follows almost the same steps as the proof of Corollary 9 except that we now use the Lyapunov function $W(e, \xi)=\varepsilon|e|+|\xi|$ that was used in Proposition 4. Note that in this case we have for almost all $e$ and $\xi$ that:

$$
\left|\frac{\partial W(e, \xi)}{\partial e}\right| \leq \varepsilon ; \quad\left|\frac{\partial W(e, \xi)}{\partial \xi}\right| \leq 1
$$

Using the fact that $\|A\| \leq \sqrt{n}\|A\|_{\infty}$, we can write for almost all $(e, \xi)$

$$
\begin{aligned}
& \left\langle\frac{\partial W(e, \xi)}{\partial(e, \xi)},\left(A e,\|A\|_{\infty} \xi\right)\right\rangle \leq\left|\frac{\partial W(e, \xi)}{\partial e}\right||A e| \\
& +\left|\frac{\partial W(e, \xi)}{\partial \xi}\right|\|A\|_{\infty}|\xi| \leq \varepsilon\|A\||| e\left|+\|A\|_{\infty}\right| \xi \mid \\
& \leq \max \left\{\|A\|,\|A\|_{\infty}\right\} W(e, \xi) \leq \sqrt{n}\|A\|_{\infty} W(e, \xi)
\end{aligned}
$$

and the proof can be completed in the same manner as before.
Remark 5: The formula for MATI in Corollary 11 for QCS is more conservative than the bound in [13] given by

$$
\tau^{*}=\frac{1}{\|A\|_{\infty}} \ln (N) .
$$

Indeed, note that $\varepsilon$ in (76) can be arbitrarily small and as $\varepsilon \rightarrow 0$ the formula (76) becomes

$$
\tau^{*}=\frac{1}{\sqrt{n}\|A\|_{\infty}} \ln (N) .
$$

The reason for this is that in the proof of Corollary 11 we used the Euclidean norm of $e$ in the Lyapunov function $W=\varepsilon|e|+$ $|\xi|$. If, on the other hand, we use the Lyapunov function $\widetilde{W}:=$ $\varepsilon\|e\|_{\infty}+|\xi|$ then we can recover the bound from [13]. Indeed, by (46) and Assumption 1, we have

$$
\left\|e^{+}\right\|_{\infty} \leq \frac{1}{N}|\xi|
$$

and using the proof similar to the one presented in Proposition 4 , we obtain that item ii) of Theorem 1 holds with $\widetilde{W}$, where $a_{1}=\min \{\varepsilon / \sqrt{n}, 1\}, a_{2}=1+\varepsilon, \rho=\frac{1+\varepsilon}{N}, \varepsilon \in(0, N-1)$. Moreover, using Hölder's inequality we have ${ }^{6}$

$$
\begin{aligned}
& \left\langle\frac{\partial \widetilde{W}}{\partial(e, \xi)},\left(A e,\|A\|_{\infty} \xi\right)\right\rangle \leq\left\|\frac{\partial \widetilde{W}}{\partial e}\right\|_{1}\|A e\|_{\infty} \\
& \quad+\left|\frac{\partial \widetilde{W}}{\partial \xi}\right|\|A\|_{\infty}|\xi| \leq\|A\|_{\infty}\left(\varepsilon\|e\|_{\infty}+|\xi|\right)=\|A\|_{\infty} W .
\end{aligned}
$$

Hence, item iii) of Theorem 1 holds with $L=\|A\|_{\infty}$ and $c=0$. Substituting these numbers into (13), we obtain

$$
\tau^{*}=\frac{1}{\|A\|_{\infty}} \ln \left(\frac{N}{\varepsilon d_{1} N+1}\right)
$$

and we recover (77) by letting $\varepsilon \rightarrow 0$. This illustrates the fact that the choice of Lyapunov function for the protocol does have an impact on the formula for MATI and exploiting this flexibility in particular cases to obtain the least conservative bounds on MATI is an option that the designer should consider.

\section{Emulation of NQCS With "Box" Protocols}

In this section we consider a class of linear NQCS whose plant dynamics are given by (68). We use the same update for $\hat{x}$ between transmission instants and, hence, the error $e$ evolves according to (74). We will consider the NQCS for the two protocols given in Section IV-C. In order to specify the $\xi$-dynamics we write the error dynamics in more detail

$$
\dot{e}_{i}=A_{i i} e_{i}+\sum_{j \neq i} A_{i j} e_{j}, \quad i=1, \ldots, \ell
$$

where the indices $i=1,2, \ldots, \ell$ are consistent with the decomposition of the vector $e=\left(e_{1}, e_{2}, \ldots, e_{\ell}\right)$ that will be used in the time-scheduling protocol; the matrices $A_{i j}$ form a partition of the matrix $A$ that is consistent with the above partition of the error vector $e$. As before, we assume that at any time instant $t_{i}$ a quantized value of only one $x_{j}, j \in\{1,2, \ldots, \ell\}$ is transmitted over the network. In order to define our quantization and the

${ }^{6}$ Note that $\|\partial \widetilde{W} / \partial e\|_{1}=\|(0, \ldots, 1, \ldots, 0)\|_{1}=1$ almost everywhere. 
corresponding quantization protocol, we need to generate the auxiliary variable $\xi \in \mathbb{R}_{\geq 0}^{\ell}$

$$
\dot{\xi}=A_{\xi} \xi \quad \forall t \in\left[t_{i-1}, t_{i}\right]
$$

and $A_{\xi}$ is chosen appropriately so that Assumption 2 holds. For example, we can define (78) via

$$
\dot{\xi}_{i}=\left\|A_{i i}\right\|_{\infty} \xi_{i}+\sum_{j \neq i}\left\|A_{i j}\right\|_{\infty} \xi_{j}, \quad i=1, \ldots, \ell
$$

where the initial data satisfies $\left\|e_{i}(0)\right\|_{\infty} \leq \xi_{i}(0) \forall i$. Then it is easy to show, using Bellman-Gronwall lemma and the arguments used to prove the standard comparison principle, that Assumption 2 holds. An alternative method is to use the vector comparison lemmas (see, for instance, [24]). If the matrix $A$ has some structure, such as block-diagonal, then (79) simplifies (cf. [6], [26]).

We apply Theorem 1 to the system (68), (74), (78) with different UGES protocols.

1) RR Protocol With Quantization: A direct consequence of UGES of protocol (52) is the following:

Corollary 12: Consider the NQCS (68), (74), (78) with (1) and the protocol (52). Suppose that $K$ is designed so that $A+$ $B K$ is Hurwitz. Then, the system is UGES if MATI satisfies $\tau \in\left(0, \tau^{*}\right)$, where

$$
\tau^{*}:=\frac{1}{\max \left\{\sqrt{\ell}\|A\|, \widetilde{a}\left\|A_{\xi}\right\|\right\}} \ln \left(\frac{1}{\rho}\right)
$$

where $\rho$ comes from Proposition 5 and $\widetilde{a}=\sqrt{\frac{N^{2} \ell}{N^{2}-1}}$.

Proof of Corollary 12: First note that $W(i, e, \xi)=$ $\varepsilon V(i, e)+U(i, \xi)$ where $V$ and $U$ are given in the proof of Proposition 5. Using Lemmas 2 and 3 in Appendix we have that the following holds for almost all $e$ and $\xi$ :

$$
\left|\frac{\partial W}{\partial e}\right| \leq \varepsilon \sqrt{\ell} ; \quad\left|\frac{\partial W}{\partial \xi}\right| \leq \widetilde{a} .
$$

Using this and the fact that $|e| \leq V(i, e)$ and $|\xi| \leq U(i, \xi)$, we can write

$$
\left\langle\frac{\partial W}{\partial(e, \xi)},\left(A e, A_{\xi} \xi\right)\right\rangle \leq\left|\frac{\partial W}{\partial e}\right|\left\|A|||e|+\left|\frac{\partial W}{\partial \xi}\right|\right\| A_{\xi}|| \xi \mid
$$$$
\leq \varepsilon \sqrt{\ell}\left\|A \left|\left\|e\left|+\widetilde{a}\left\|A_{\xi}\right\|\right| \xi \mid \leq \max \left\{\sqrt{\ell}\|A\|, \widetilde{a}\left\|A_{\xi}\right\|\right\} W(i, e, \xi)\right.\right.\right. \text {. }
$$

The proof is completed by using formula (13) with $L=\max \left\{\sqrt{\ell}\|A\|, \widetilde{a}\left\|A_{\xi}\right\|\right\}$ and $c=0$ with $\rho$ coming from Proposition 5.

2) TOD Protocol With Quantization: The proof of the following result follows the same steps using the Lyapunov function from Proposition 6 and it is omitted.

Corollary 13: Consider the NQCS (68), (74), (78) with (1) and the protocol (57). Suppose that $K$ is designed so that $A+$ $B K$ is Hurwitz. Then, the system is UGES if MATI satisfies $\tau \in\left(0, \tau^{*}\right)$, where

$$
\tau^{*}:=\frac{1}{\max \left\{\|A\|,\left\|A_{\xi}\right\|\right\}} \ln \left(\frac{1}{\rho}\right)
$$

and $\rho$ comes from Proposition 6.

Remark 6: We revisit the protocol (59)-(61) in Remark 2 that can be used to illustrate the flexibility of our approach.
To this end, we note that Theorem 1 was proved in [19] by showing that items ii)-iv) of the Theorem imply that the subsystem (6), (7) has a finite gain ISS gain from $x$ to $z$. Moreover, this gain, denoted as $\kappa_{\tau}(s):=\gamma_{e}(\tau) \cdot s$, depends on MATI and we have that $\lim _{\tau \rightarrow 0} \gamma_{e}(\tau)=0$. Since in item i) of Theorem 1 we assume a finite ISS gain from $z$ to $x$ of the system (5), denoted as $\gamma$, we can apply the small-gain theorem and calculate $\tau^{*}$ for which the small-gain condition holds: $\gamma \cdot \gamma_{e}(\tau)<1$, $\forall \tau \in\left(0, \tau^{*}\right)$. This is exactly how the (13) is obtained. While we cannot use directly Theorem 1 for this example, we can still use the above small-gain argument to prove stability with the protocol (59)-(61) under Assumption 2. (Note that the initial condition (62) violates Assumption 2.)

We begin by showing that the $\xi$-subsystem is UGES in the sense of our Definition 2. This can be proved by using $W(\xi)=|\xi|$. From Lemma 1 in Appendix we have that: $W\left(\xi^{+}\right) \leq \sqrt{\frac{N^{2} \ell-N^{2}+1}{N^{2} \ell}} W(\xi)$. It is not hard to show that for linear systems also item iii) holds with $L=\left\|A_{\xi}\right\|$ and $c=0$. Hence, the $\xi$-subsystem is UGES (ISS with zero gain) for all $\tau \in\left(0, \tau^{*}\right)$ where

$$
\tau^{*}=\frac{1}{\left\|A_{\xi}\right\|} \ln \left(\sqrt{\frac{N^{2} \ell}{N^{2} \ell-N^{2}+1}}\right) .
$$

Moreover, we discussed already how one can adjust the parameters in the protocol so that Assumption 2 holds. Hence, we can conclude that for the same value of MATI the $(e, \xi)$-subsystem is UGES for all initializations that satisfy Assumption 2. Hence, the system behaves like a cascade of the UGES $(e, \xi)$-subsystem and a linear ISS $x$-subsystem, and we can conclude that the overall NQCS is UGES. We note that in the preceding more systematic approach, which relies on an off-the-shelf application of Theorem 1, Assumption 2 was not used explicitly. Instead, we used its consequence (49) which is in principle more general. $\square$

\section{Emulation of NQCS With Zoom Protocols}

In this section we consider NQCS of the form (37) in which the first two equations are linear and given by (68), (69). We consider the zoom protocols for NQCS whose stability properties were analyzed in Sections IV-D-1 and 2. Using similar arguments as before, we can obtain the following two results that are stated without proofs.

1) RR With Zoom Protocols:

Corollary 14: Consider the NQCS (37) in which the first two equations are linear and given by (68), (69) and the protocol is given by (64) with (40). Suppose that $K$ is designed so that $A+B K$ is Hurwitz and let $\kappa(s)=\gamma \cdot s, \gamma \geq 0$ be the ISS gain from $e$ to $x$ for the system (68). Then, the system is UGES if MATI satisfies $\tau \in\left(0, \tau^{*}\right)$, where $\tau^{*}$ is given by (13) with $L=\sqrt{\ell}\|B K\|$ and $c=\varepsilon \sqrt{\ell}\|A+B K\|$ and $\rho, a_{1}$ and $\varepsilon$ come from Proposition 7.

2) TOD With Zoom Protocols:

Corollary 15: Consider the NQCS (37) in which the first two equations are linear and given by (68), (69) and the protocol is given by (64) with (43). Suppose that $K$ is designed so that $A+B K$ is Hurwitz and let $\kappa(s)=\gamma \cdot s, \gamma \geq 0$ be the ISS gain from $e$ to $x$ for the system (68). Then, the system is UGES if MATI satisfies $\tau \in\left(0, \tau^{*}\right)$, where $\tau^{*}$ is given by (13) with 
$L=\|B K\|$ and $c=\varepsilon\|A+B K\|$ and $\rho, a_{1}$ and $\varepsilon$ come from Proposition 8.

\section{CONCLUSION}

In this paper, we unified results on emulation for QCS and NCS by generalizing recently reported results for NCS in [19]. A central issue in our approach is proving stability properties of the corresponding quantization/time-scheduling protocols. We illustrated how this can be done for several representative protocols. Our approach is amenable to various generalizations, modifications and extensions, such as considering: persistently exciting protocols (see [24]) that are essential in wireless networked control systems; stochastic protocols and plants (see [7], [23]); UGAS protocols [20]; and ISS protocols (see for instance the class of "zoom" protocols in [12], discussed from this ISS viewpoint in [18]).

\section{APPENDIX}

Lemma 1: The protocol (60), (61) is UGES with the Lyapunov function $W(\xi)=|\xi|$. In particular, we can take $a_{1}=$ $a_{2}=1$ and $\rho=\sqrt{\frac{N^{2} \ell-N^{2}+1}{N^{2} \ell}}$.

Proof of Lemma 1: The following holds by direct calculations, where in the last step we use (63):

$$
\begin{aligned}
W\left(\xi^{+}\right) & =\sqrt{\sum_{i \neq s} \xi_{i}^{2}+\frac{1}{N^{2}} \xi_{s}^{2}}=\sqrt{|\xi|^{2}-\frac{N^{2}-1}{N^{2}} \xi_{s}^{2}} \\
& \leq \sqrt{\left(1-\frac{N^{2}-1}{N^{2} \ell}\right)|\xi|^{2}}=\sqrt{\frac{N^{2} \ell-N^{2}+1}{N^{2} \ell}} W(\xi)
\end{aligned}
$$

Lemma 2: Consider the protocol (39), (40) and let $W(i, e)=\sqrt{V(i, e)}$ where $V$ is given by (41). Then, $|\partial W(i, e) / \partial e| \leq \sqrt{\ell}$ for almost all $e$ and all $i$. Moreover, $W$ is globally Lipschitz with the Lipschitz constant equal to $\sqrt{\ell}$.

Proof of Lemma 2: First, we note that since the protocol is dead-beat stable, $V$ in (41) can be written as follows $V(i, e)=$ $\sum_{i=1}^{\ell} a_{j}(i)\left|e_{i}\right|^{2}$, where we have that

$$
a_{j}(i) \leq \ell \quad \forall j \in\{1, \ldots, \ell\}, \forall i .
$$

Hence, we have the following:

$$
\begin{aligned}
\left|\frac{\partial W(i, e)}{\partial e}\right| & =\left|\frac{\left(a_{1}(i) e_{1}, a_{2}(i) e_{2}, \ldots, a_{\ell}(i) e_{\ell}\right)}{\sqrt{\sum_{j} a_{j}(i)\left|e_{j}\right|^{2}}}\right| \\
& =\frac{\sqrt{\sum_{j} a_{j}^{2}(i)\left|e_{j}\right|^{2}}}{\sqrt{\sum_{j} a_{j}(i)\left|e_{j}\right|^{2}}} \leq \sqrt{\ell} \frac{\sqrt{\sum_{j} a_{j}(i)\left|e_{j}\right|^{2}}}{\sqrt{\sum_{j} a_{j}(i)\left|e_{j}\right|^{2}}}=\sqrt{\ell} .
\end{aligned}
$$

The Lipschitzness of $V$ follows from the Lebourg's Lipschitz Mean Value Theorem [3, Theorem 2.3.7].

Lemma 3: Consider the protocol consisting of the $\xi$-subsystem in (52) and let $U(i, \xi)=\sqrt{\widetilde{U}(i, \xi)}$, where $\widetilde{U}(i, \xi)$ comes from (53). Then, $|\partial U(i, \xi) / \partial \xi| \leq \sqrt{\frac{N^{2} \ell}{N^{2}-1}}=: \widetilde{a}$ for almost all $\xi$ and all $i$. Moreover, $U$ is globally Lipschitz with the Lipschitz constant equal to $\widetilde{a}$.

Proof of Lemma 3: First note that by directly inspecting $\phi_{\xi}(k, i, \xi)$ we can see that there exist $a_{j}(k, i) \in \mathbb{N}$ for $j \in\{1,2, \ldots, \ell\}$ and $k, i \in \mathbb{N}, k \geq i$ such that $U(i, \xi)=$ $\sqrt{\sum_{j=1}^{\ell} \sum_{k=i}^{\infty} a_{j}(k, i) \xi_{j}^{2}}$, where for any $j \in\{1,2, \ldots, \ell\}$, arbitrary $i \in \mathbb{N}$ and all $k \in[i+p \ell, i+(p+1) \ell-1], p=0,1, \ldots$ we have that $a_{j}(k, i) \leq\left(1 / N^{2}\right)^{p}$ and we can write for any $j \in\{1,2, \ldots, \ell\}$ that

$$
\sum_{k=i}^{\infty} a_{j}(k, i) \leq \ell \sum_{p=0}^{\infty}\left(\frac{1}{N^{2}}\right)^{p}=\frac{\ell}{1-\frac{1}{N^{2}}}=\frac{N^{2} \ell}{N^{2}-1} .
$$

Hence, the following holds for almost all $\xi$ and all $i$ :

$$
\begin{aligned}
\left|\frac{\partial U(i, \xi)}{\partial \xi}\right| & =\left|\frac{\left(\sum_{k=i}^{\infty} a_{1}(k, i) \xi_{1}, \ldots, \sum_{k=i}^{\infty} a_{\ell}(k, i) \xi_{\ell}\right)}{\sqrt{\sum_{j=1}^{\ell} \sum_{k=i}^{\infty} a_{j}(k, i) \xi_{j}^{2}}}\right| \\
& =\frac{\sqrt{\sum_{j=1}^{\ell} \sum_{k=i}^{\infty} a_{j}^{2}(k, i) \xi_{j}^{2}}}{\sqrt{\sum_{j=1}^{\ell} \sum_{k=i}^{\infty} a_{j}(k, i) \xi_{j}^{2}}} \\
& \leq \sqrt{\frac{N^{2} \ell}{N^{2}-1}} \frac{\sqrt{\sum_{j=1}^{\ell} \sum_{k=i}^{\infty} a_{j}(k, i) \xi_{j}^{2}}}{\sqrt{\sum_{j=1}^{\ell} \sum_{k=i}^{\infty} a_{j}(k, i) \xi_{j}^{2}}}=\sqrt{\frac{N^{2} \ell}{N^{2}-1}}=\widetilde{a} .
\end{aligned}
$$

The Lipschitzness of $U$ follows from the Lebourg's Lipschitz Mean Value Theorem [3, Theorem 2.3.7].

\section{REFERENCES}

[1] R. W. Brockett and D. Liberzon, "Quantized feedback stabilization of linear systems," IEEE Trans. Automat. Control, vol. 45, no. 7, pp. 1279-1289, Jul. 2000.

[2] T. Chen and B. A. Francis, "Input-output stability of sampled-data systems," IEEE Trans. Automat. Control, vol. 36, no. 1, pp. 50-58, Jan. 1991.

[3] F. H. Clarke, Optimization and Non-Smooth Analysis. Philadelphia, PA: SIAM, 1990, vol. 5, Classics in applied mathematics.

[4] D. F. Delchamps, "Stabilizing a linear system with quantized state feedback," IEEE Trans. Automat. Control, vol. 35, no. 8, pp. 916-924, Aug. 1990.

[5] H. A. Edwards, Y. Lin, and Y. Wang, "On Input-to-State Stability for Time Varying Nonlinear Systems," in Proc. 29th IEEE Conf. Decision Control, 2000, pp. 3501-3506.

[6] J. P. Hespanha, A. Ortega, and L. Vasudevan, "Towards the control of linear systems with minimum bit-rate," in Proc. 15th Int. Symp. Math. Theory Networks Syst. (MTNS), 2002, [CD ROM].

[7] J. P. Hespanha and A. R. Teel, "Stochastic impulsive systems driven by renewal processes," in Proc. Int. Symp. Math. Theory Networks Syst. (MTNS), 2006, [CD ROM].

[8] D. Hristu and K. Morgansen, "Limited communication control," Syst. Control Lett, vol. 37, pp. 193-205, 1999.

[9] H. Ishii and B. Francis, "Stabilization with control networks," Automatica, vol. 38, pp. 1745-1751, 2002.

[10] Z.-P. Jiang, A. R. Teel, and L. Praly, "Small-gain theorem for ISS systems and applications," Math. Control Signals Syst., vol. 7, pp. 95-120, 1994

[11] S. Lall and G. Dullerud, "An LMI solution to the robust synthesis problem for multi-rate sampled-data systems," Automatica, vol. 37, pp. 1909-1922, 2001. 
[12] D. Liberzon, "Hybrid feedback stabilization of systems with quantized signals," Automatica, vol. 39, pp. 1543-1554, 2003.

[13] D. Liberzon, "On stabilization of linear systems with limited information," IEEE Trans. Automat. Control, vol. 48, no. 2, pp. 304-307, Feb. 2003.

[14] D. Liberzon and J. P. Hespanha, "Stabilization of nonlinear systems with limited information feedback," IEEE Trans. Automat. Control, vol. 50, no. 6, pp. 910-915, Jun. 2005.

[15] D. Liberzon and D. Nešić, "Input-to-state stabilization of linear systems with quantized state measurements," IEEE Trans. Automat. Control, vol. 52, no. 5, pp. 767-781, May 2007.

[16] Q. Ling and M. D. Lemmon, "Stability of quantized control systems under dynamic bit assignment," IEEE Trans. Automat. Control, vol. 50, no. 5, pp. 734-740, May 2005.

[17] G. N. Nair and R. J. Evans, "Stabilizability of stochastic linear systems with finite feedback data rates," SIAM J. Control Optim, vol. 43, pp. 413-436, 2004.

[18] D. Nešić and D. Liberzon, "A small-gain approach to stability analysis of hybrid systems," in Proc. 44th IEEE Conf. Decision Control, 2005, pp. 5409-5414.

[19] D. Nešić and A. R. Teel, "Input-output stability properties of networked control systems," IEEE Trans. Automat. Control, vol. 49, no. 10, pp. 1650-1667, Oct. 2004.

[20] D. Nešić and A. R. Teel, "Input to state stability of networked control systems," Automatica, vol. 40, pp. 2121-2128, 2004.

[21] I. R. Petersen and A. V. Savkin, "Multi-rate stabilization of multivariable discrete-time linear systems via a limited capacity communication channel," in Proc. 40th IEEE Conf. Decision Control, 2001, pp. 304-309.

[22] E. D. Sontag, "Smooth stabilization implies coprime factorization," IEEE Trans. Automat. Control, vol. 34, no. AC-4, pp. 435-443, Apr. 1989.

[23] M. Tabbara and D. Nešić, "Input-output stability of networked control systems with stochastic protocols and channels," IEEE Trans. Automat. Control, vol. 53, no. 5, pp. 1160-1175, Jun. 2008.

[24] M. Tabbara, D. Nešić, and A. R. Teel, "Stability of wireless and wireline networked control systems," IEEE Trans. Automat. Control, vol. 52, no. 9, pp. 1615-1630, Sep. 2007.

[25] S. Tatikonda, "Some scaling properties of large distributed control systems," in Proc. 42nd IEEE Conf. Decision Control, 2003, pp. 3142-3147.

[26] S. Tatikonda and S. K. Mitter, "Control under communication constraints," IEEE Trans. Automat. Control, vol. 49, no. 7, pp. 1056-1068, Jul. 2004.

[27] G. C. Walsh, O. Beldiman, and L. G. Bushnell, "Asymptotic behavior of nonlinear networked control systems," IEEE Trans. Automat. Control, vol. 46, no. 7, pp. 1093-1097, Jul. 2001.

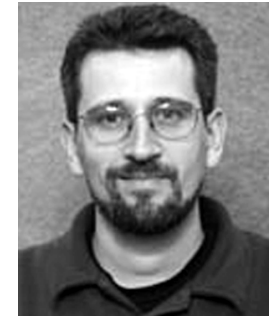

Dragan Nešić (S'96-M'02-SM'02-F'08) received the B.E. degree in mechanical engineering from The University of Belgrade, Belgrade, Yugoslavia in 1990 and the Ph.D. degree from Systems Engineering, RSISE, Australian National University, Canberra, in 1997.

$\mathrm{He}$ is a Professor in the Department of Electrical and Electronic Engineering (DEEE), University of Melbourne, Victoria, Australia. From 1997 to 1999 , he held postdoctoral positions at DEEE; CESAME, Universite Catholique de Louvain, Louvain la Neuve, Belgium; and ECE, University of California, Santa Barbara. Since February 1999, he has been with The University of Melbourne. He is an Associate Editor for Automatica, Systems and Control Letters, and the European Journal of Control. His research interests include networked control systems, discrete-time, sampled-data and continuous-time nonlinear control systems, input-to-state stability, extremum seeking control, applications of symbolic computation in control theory, hybrid control systems, and so on.

Dr. Nešić received the Humboldt Research Fellowship from the Alexander von Humboldt Foundation in 2003 and the Australian Professorial Fellowship (2004-2009) from the Australian Research Council. He is a Fellow of IEAust, an Associate Editor for the IEEE TRANSACTIONS ON AUTOMATIC CONTROL, and a Distinguished Lecturer of CSS, IEEE (2008-2010).

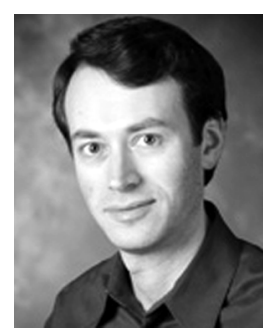

Daniel Liberzon (M'98-SM'04) was born in the former Soviet Union on April 22, 1973. He received the M.S. degree from the Department of Mechanics and Mathematics, Moscow State University, Moscow, Russia, in 1993 and the Ph.D. degree in mathematics from Brandeis University, Waltham, MA, in 1998.

Following a postdoctoral position in the Department of Electrical Engineering, Yale University, New Haven, CT, from 1998 to 2000, he joined the University of Illinois at Urbana-Champaign, where he is now an Associate Professor in the Electrical and Computer Engineering Department and a Research Associate Professor in the Coordinated Science Laboratory. He wrote Switching in Systems and Control (Boston, MA: Birkhauser, 2003) and authored or coauthored over 30 journal articles on the following topics. His research interests include nonlinear control theory, analysis and synthesis of switched systems, control with limited information, and uncertain and stochastic systems.

Dr. Liberzon received the IFAC Young Author Prize and the NSF CAREER Award, both in 2002, the Donald P. Eckman Award from the American Automatic Control Council in 2007, and the Xerox Award for Faculty Research from the UIUC College of Engineering also in 2007. Since 2007, he has served as an Associate Editor for the IEEE TRANSACTIONS ON AUTOMATIC CONTROL. 